\title{
Origin of exotic clasts in the Central-Southern Apennines: clues to the Cenozoic fold-and-thrust collisional belt in the Central Mediterranean area
}

\author{
S. ARRAGONI*†, L. P. FERNÁNDEZ \\ \& F. SALVINI* \\ * Dipartimento di Scienze, Sezione di Geologia, Università di Roma Tre, L.go San Leonardo \\ Murialdo 1, 00146 Roma, Italy \\ †Departamento de Geología, Universidad de Oviedo, C./ Jesús Arias de Velasco s/n, 33005 Oviedo, Spain
}

(Received 29 October 2016; accepted 21 September 2017; first published online 30 October 2017)

\begin{abstract}
The Central-Southern Apennines are the result of the collision between Europe and Africa. Despite the volume of existing literature, many problems remain unsolved such as the presence of Tertiary conglomerates containing exotic basement clasts. The lack of basement rocks in the CentralSouthern Apennines implies that the origin of these clasts has to be sought in areas where the basement is extensively exposed. These include the Calabro-Peloritani arc and the Sardinia-Corsica block, which in Cenozoic time were connected to the Central-Southern Apennines. In this work we present the results of sedimentary, geochemical and petrographic analyses performed on the exotic basementderived clasts. These analyses include lithological, major- and minor-element and rare Earth element compositions which are compared to analogous rocks from Calabria and Sardinia basements. Results indicate Eastern Sardinia as the primary source area for the studied conglomeratic units, linking the Central-Southern Apennines sedimentary cover to the Mesozoic carbonates of Eastern Sardinia prior to the opening of Tyrrhenian Sea. The Cilento unit (Campania) was directly fed by an uplifting Cenozoic orogen, and the Filettino, Gavignano (Latium) and Ariano Irpino (Campania) units were produced by the successive reworking of 'Cilento-like' sedimentary units. These results may imply that part of the Central-Southern Apennines represented a portion of the European margin of the Tethys.
\end{abstract}

Keywords: Central-Southern Apennines, Sardinia, fold-and-thrust belt, European sedimentary wedge, conglomerates

\section{Introduction}

The Central Mediterranean is a very complicated yet interesting geological puzzle. At the regional scale, the development of this basin and surrounding mountain chains is related to the interaction between the African and European plates since Late Cretaceous time (e.g. Biju-Duval et al. 1974; Laubscher \& Bernoulli, 1977; Vai \& Martini, 2001). Nonetheless, the single features are difficult to frame into a 'simple' collisional geodynamic context.

Scattered fragments of an ancient collisional chain that once extended from Gibraltar to the Alps have been recognized in the islands of Sardinia and Corsica (e.g. Alvarez, 1976, 1991), separated from the current French-Spanish coast in early Miocene time (Burdigalian-Langhian; Speranza et al. 2002) by the opening of the Liguro-Provençal Basin. In particular, on the eastern coast of Sardinia (Golfo di Orosei area), remnants of a $\mathrm{W}$-verging (in present-day coordinates) fold-and-thrust belt have been found (Arragoni et al. 2016), resulting from the deformation of the European sedimentary wedge and its

$\dagger$ Author for correspondence: simone.arragoni@uniroma3.it basement in Cenozoic time. Despite their noteworthy geological significance, these small relics of the collisional belt cannot account for the entire European palaeo-sedimentary wedge; an important part of this palaeogeographic domain is apparently missing.

Furthermore, most collisional chains from around the world are characterized by double vergence, for example: the Rocky Mountains (Bally, Gordy \& Stewart, 1966; Fitz-Diaz, Hudleston \& Tolson, 2011); the Alps (Schmid et al. 1996; Lardeaux et al. 2006; Lammerer et al. 2008); and the Himalaya (Butler, 1986; Acton et al. 2010). As evidenced by Arragoni et al. (2016), the Eastern Sardinia fold-and-thrust belt shows a westwards vergence related to the westwards subduction (in present-day coordinates) of the African Plate beneath the European Plate, and the opposite verging side may be heavily hidden within the Apennines by the successive post-Oligocene Tyrrhenian Sea opening and Apennine chain formation.

The Eastern Sardinia mountain belt has been deeply eroded since Eocene time. The Mesozoic sedimentary cover was completely removed in many places, leaving the Palaeozoic basement exposed together with the deeper flat-over-flat sectors of the regional thrusts (sole thrusts). This erosional phase implies the production of 
a huge amount of sediments (both carbonate and silicoclastic) and raises some questions: which are the Cenozoic sedimentary units that record this event? Where are these units located now?

The opening of the Liguro-Provençal and Tyrrhenian Sea basins, which disrupted and translated the former Alpine collisional chain in Neogene time, determined the formation of the Apennines. Here we will use the term 'Central-Southern Apennines' to indicate the portion of the Apennines between the AnconaAnzio line (Parotto \& Praturlon, 1975; reinterpreted as structural element the Olevano-Antrodoco line by Salvini \& Vittori, 1982) and the Calabria-Peloritani block.

This oblique chain (with respect to the presentday orientation of the zone of convergence between Africa and Europe) derives from the deformation of the Meso-Cenozoic sedimentary units (mainly carbonates) detached from their basement (e.g. Vai \& Martini, 2001). According to the most accepted interpretations (e.g. Carmignani et al. 2001a; Barchi et al. 2001; Molli, 2008; Rossetti et al. 2015) the Northern Apennines resulted from the deformation of the African margin; a sequence of palaeogeographic domains can be reconstructed, from the ancient continental passive margin represented by the UmbriaMarche and Tuscany successions, to the Tethys oceanic domain represented by the ophiolite-bearing Liguride units. These oceanic units are tectonically superposed on the whole African palaeo-passive margin units.

In the last decades, a number of high-quality studies have been performed in the Central-Southern Apennines with the development of several models (e.g. Scandone, 1967, 1975; Ogniben, 1969, 1972; D'Argenio, Pescatore \& Scandone, 1973; Pescatore \& Ortolani, 1973; Mostardini \& Merlini, 1986; Sgrosso, 1986; Patacca et al. 1990; Vai \& Martini, 2001; Vezzani, Festa \& Ghisetti, 2010). However, due to the complex geological evolution of this area, the geodynamic significance and attribution of deformed units is still debated and a complete sequence of palaeogeographic domains is more difficult to reconstruct. In this area the Liguride units occur both (mostly) under and over the Meso-Cenozoic sedimentary cover (e.g. Majella Mountains: SGN, 1970; Volsci Range and Latina Valley: Angelucci, Devoto \& Farinacci, 1963; Angelucci \& Devoto, 1966; SGN, 1967; Cilento: Monaco \& Tortorici, 1995; Catalano et al. 2004).

A crucial open question in the Central-Southern Apennines is the presence of widespread conglomeratic units containing igneous and metamorphic basement clasts whose origin and significance are still controversial. These include many different scattered sedimentary units cropping out from the Filettino area (NE of Rome, Latium) to the Cilento region (SE of Naples, Campania). The presence of basement clasts in these conglomerates is a striking feature already noted and discussed by the first researchers who stud- ied the geology of the Central-Southern Apennines (e.g. Viola, 1898; Sacco, 1910; Beneo, 1943). In the existing geological maps of the region they are usually reported as 'puddinghe, talora con elementi di rocce intrusive (graniti, porfidi) ed altre rocce estranee alla regione' ('clast-supported conglomerates, sometimes with clasts of intrusive rocks - granites, porphyries - and other exotic rocks'; SGN, 1939), 'puddinghe poligeniche' ('polygenic clast-supported conglomerates'; SGN, 1963), 'Conglomerati a matrice prevalente, con elementi di rocce cristalline e calcaree' ('matrix-supported conglomerates, with clasts of crystalline and calcareous rocks'; SGN, 1969) or 'Conglomerati stratificati, a ciottoli grossolani di natura prevalentemente estranea alla serie locale, ('well-bedded conglomerates, with coarse-grained clasts of prevailingly exotic origin'; SGN, 1975).

Despite the apparently local significance of these deposits, their correct interpretation could bear dramatic consequences for the geological interpretation of the whole Central Mediterranean area. As already discussed the sedimentary cover of the Central-Southern Apennines lacks its basement and outcrops of metamorphic or igneous lithologies are absent, except in the Calabro-Peloritani arc (Bonardi et al. 2001) and in very small outcrops of basement rocks (e.g. the Zannone Island, De Rita et al. 1986; Pantosti \& Velonà, 1986) and metamorphosed limestones, probably related to hydrothermal alteration due to the presence of regional-scale faults (e.g. the Mondragone yellow marble, Di Girolamo et al. 2000). The source area for these exotic clasts therefore has to be searched for in places where the basement was extensively exposed at the time of deposition, and also subjected to the typical peneplanation processes active in the various geodynamic environments after the tectonic activity (Cianfarra \& Salvini, 2014; Maggi, Cianfarra \& Salvini, 2016; Cianfarra \& Maggi, 2017). These include the Calabro-Peloritani arc and the SardiniaCorsica block (Maggi et al. 2012, 2014; Rossetti et al. 2015), which were physically connected to the CentralSouthern Apennines before the opening of the Tyrrhenian Sea (Langhian: Kastens \& Mascle, 1990; Sartori et al. 2004).

The recognition of specific source areas for these conglomerates allows the geological evolution of the Central-Southern Apennines to be linked to that of Sardinia, hence providing new clues for the reconstruction of the Meso-Cenozoic European sedimentary wedge.

In this work we analyse the sedimentary features of four different conglomeratic units cropping out from Filettino to Cilento. The petrographic and geochemical features of igneous clasts sampled in selected outcrops are described and compared with data taken from the existing literature about Sardinia and Calabria.

The geodynamic implications of our results are discussed in order to add new constraints for unravelling the complex Tertiary geological evolution of 


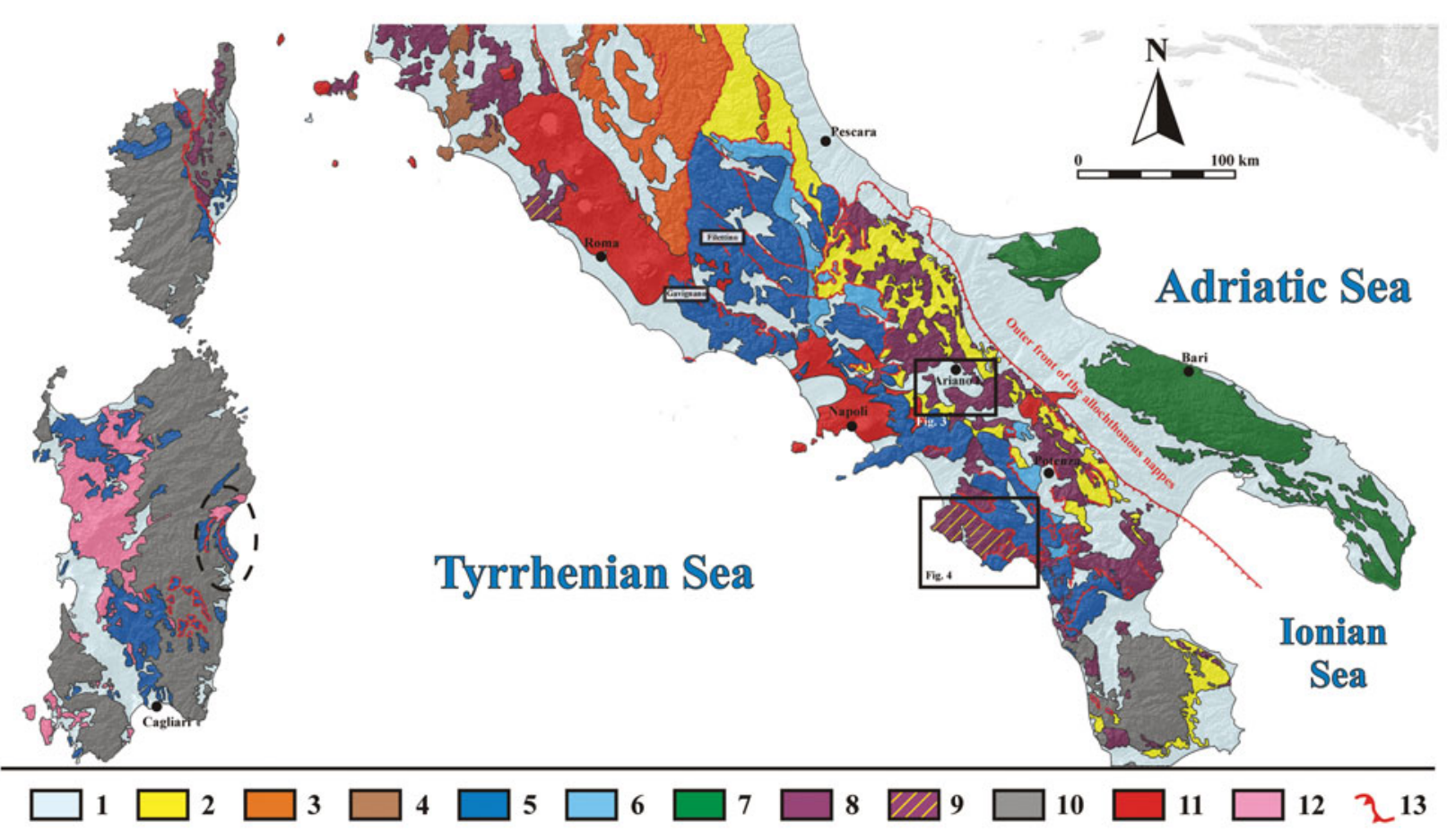

Figure 1. (Colour online) Simplified geological map of the Southern Apennines and Corsica and Sardinia Islands (modified from Bigi et al. 1992). Key to symbols: 1, Plio-Quaternary deposits (except volcanic rocks); 2, Cenozoic terrigenous units; 3, Umbria-Marche sedimentary units (Upper Triassic - Miocene); 4, Tuscany sedimentary units (Upper Triassic - Oligocene); 5, sedimentary cover of the European sedimentary wedge (mainly shallow-water limestones: Latium-Abruzzi and Campano-Lucana carbonate platforms) (Middle Triassic - Miocene); 6, Meso-Cenozoic pelagic sedimentary units of the Southern Apennines (including the LagonegresiMolisane units); 7, Apula carbonate platform (Upper Triassic - Miocene); 8, ocean-derived units, including ophiolites and varicoloured clays (Cretaceous-Eocene); 9, Pietraforte and Cilento sedimentary units (Upper Cretaceous - Miocene); 10, Palaeozoic basement units (Corsica, Sardinia and Calabria); 11, Cenozoic volcanic rocks (Italian Peninsula); 12, Cenozoic volcanic rocks (Sardinia); and 13, main thrusts. The black ellipse indicates the source area of the exotic-clast-bearing conglomerates of the Southern Apennines.

the Central-Southern Apennines and the surrounding areas.

\section{Geological framework}

The Central-Southern Apennines (Fig. 1) form part of the Apennine Chain, a long (about $1500 \mathrm{~km}$ ), arcuate collisional chain connecting the Western Alps to the Maghrebian Chain of Northern Africa (Vezzani, Festa \& Ghisetti, 2010).

The Apenninic chain still preserves tectonostratigraphic imprints of superposed events of rifting, drifting, subduction and collision related to the interaction between the African and European plates since Triassic time (e.g. Cavazza, Roure \& Ziegler, 2004; Dilek, 2006; Patacca \& Scandone, 2007). The roughly N$\mathrm{S}$ convergence between the two plates started in Late Cretaceous time (Alpine orogeny: Trümpy, 1973; Castellarin \& Transalp Working Group, 2004) and determined the closure of the Tethys Ocean and the development of the Alpine collisional chain extended from Gibraltar to the present-day Alps (Alvarez 1976, 1991), whose relics are well preserved in Eastern Sardinia (Arragoni et al. 2016) and Corsica (Maggi et al. 2012, 2014; Rossetti et al. 2015). The opening of the Tyrrhenian Sea since Langhian time (Kastens
\& Mascle, 1990; Sartori et al. 2004) determined the eastwards translation of part of the deformed MesoCenozoic sedimentary covers of the chain. These were detached from their basement to form the Apenninic orogen. This event produced the NE-directed migration of a compressional front (Boccaletti et al. 1990; Cipollari \& Cosentino, 1995; Cosentino, Cipollari \& Pipponzi, 2003), currently located in the Adriatic Sea (Favali et al. 1993; D’Agostino et al. 2008). The compressional deformation was accompanied by extension on the eastern side of the Tyrrhenian Sea and the facing Apennines (e.g. Carmignani et al. 1994; Vai \& Martini, 2001; Brogi et al. 2005; Arragoni, Martini \& Sandrelli, 2012; Martini et al. 2013).

In the Southern Apennines the tectonically superposed units include the Liguride units (CretaceousEocene: Ogniben, 1969; Knott, 1987) together with the 'varicoloured clays' (Angelucci, Devoto \& Farinacci, 1963; Angelucci \& Devoto, 1966) whose presentday structural position (e.g. Monaco \& Tortorici, 1995; Catalano et al. 2004; Tortorici, Catalano \& Monaco, 2009) does not unequivocally clarify their palaeogeographic position. This tectonic pattern determined the formation of several tectonically controlled basins in which terrigenous sequences accumulated since 

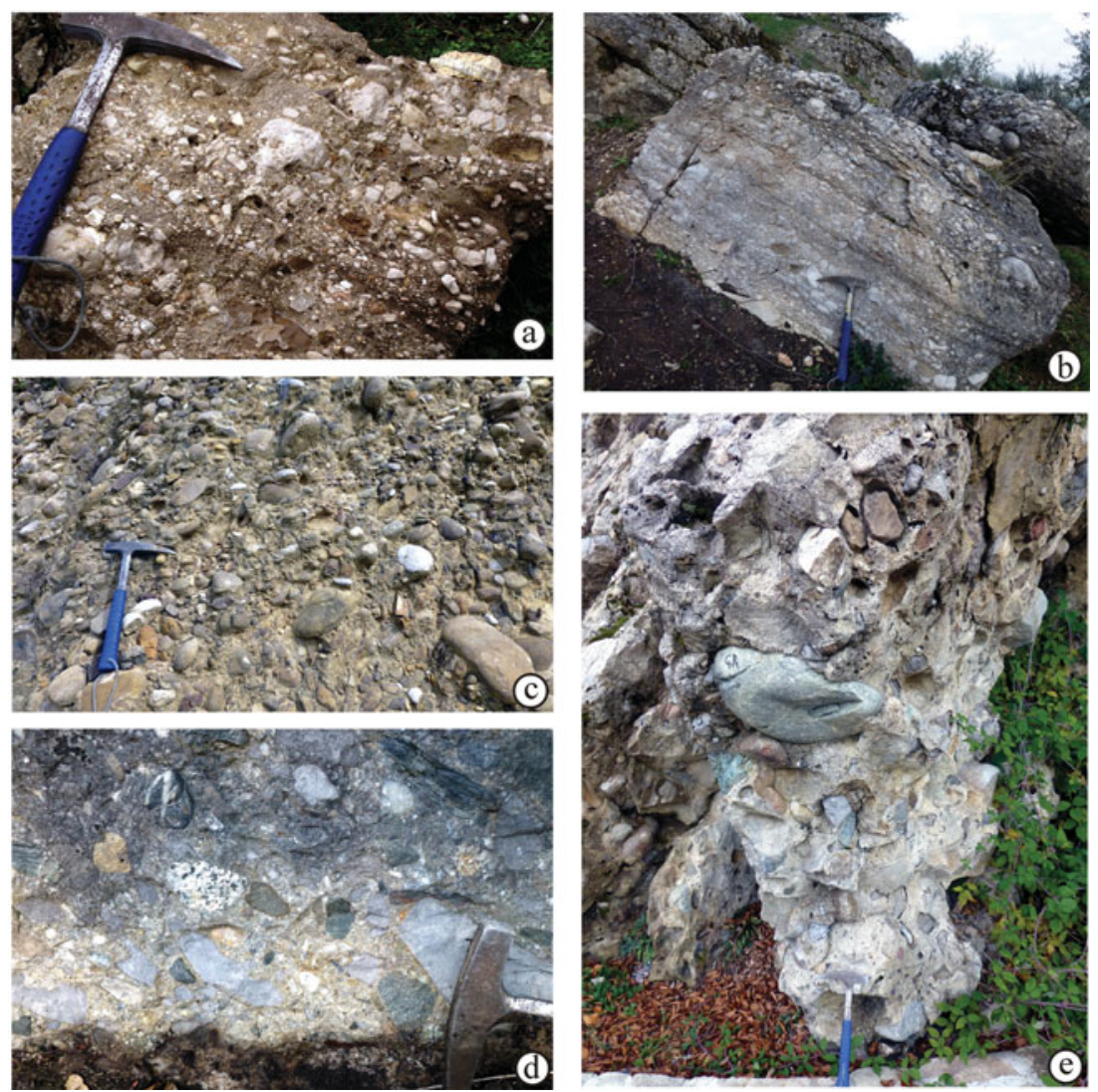

Figure 2. (Colour online) Main features of the studied conglomeratic units. (a) Filettino conglomerates; (b) Gavignano conglomerates; (c) Ariano Irpino conglomerates; (d) Cilento conglomerates; and (e) Cilento conglomerates, top of Monte Gelbison.

Cenozoic time (e.g. Martini, Sagri \& Colella, 2001; Patacca \& Scandone, 2001). The studied conglomerates were deposited prior or contemporary to these sequences.

\section{Exotic-clast-bearing conglomerates in the Central-Southern Apennines}

The Tertiary terrigenous units of the Central-Southern Apennines include a great variety of deposits representing a wide range of ages and sedimentary environments (Vezzani, Festa \& Ghisetti, 2010).

Despite the substantial lack of basement outcrops along this segment of the chain (from the AnconaAnzio line down to the Calabrian Arc), several of these terrigenous units contain crystalline and metamorphic clasts of internal (i.e. westwards in present-day coordinates) provenance (Civitelli \& Corda, 1982; Sartori, Mascle \& Amaudric du Chaffaut, 1987) whose geodynamic significance is still puzzling.

In this work we investigate four main sedimentary units scattered along the whole chain from north to south, namely: Filettino (the northernmost outcrop, $70 \mathrm{~km}$ east of Rome); Gavignano and surroundings (50 km SE of Rome); Ariano Irpino (80 km NNE of Naples); and Cilento (the southernmost and largest unit, $100 \mathrm{~km}$ SE of Naples). In the following sections these sedimentary units are referred to by the name of the main outcrop locality.

\section{3.a. Filettino}

The Filettino conglomerates form a small outcrop (about $0.5 \mathrm{~km}^{2}$ ) on the northern flank of Monte Viglio (Filettino, $41^{\circ} 55^{\prime} 26^{\prime \prime} \mathrm{N}, 13^{\circ} 22^{\prime} 32^{\prime \prime} \mathrm{E}$ ). Poor exposure conditions and the small size of the outcrop have attracted only a little interest previously (Beneo, 1943; Devoto, 1967a, b; Accordi et al. 1969), which resulted in uncertain interpretations of their origin and stratigraphic position. On the other hand, their precise dating is not so important for this study as the focus is the origin of the exotic clasts. Devoto (1967b) attributed these deposits to the Fosso Fioio Unit (Messinian), describing an unconformable base over the Upper Cretaceous units of the deformed Mesozoic carbonate platform. Nevertheless, this contact is not clearly exposed today. The unit is about $50 \mathrm{~m}$ thick and consists of thick beds (up to $1 \mathrm{~m}$ ) of polygenic conglomerates with well-rounded clasts, mainly made of shallowwater limestones, sandstones, older conglomerates and rare yellow micritic limestones. Clasts are poorly sorted, ranging from granules to cobbles, and are often aligned parallel to bedding (Fig. 2a). The sedimentation of the Filettino conglomerates can be related to mass-wasting processes, in particular to unconfined cohesionless debris flows (Nemec \& Steel 1984; Nemec et al. 1984; see also Lowe 1982).

A 'Lago Mare' environment (Orszag-Sperber, 2006) was proposed by Cosentino \& Cipollari (2012) for 
similar deposits cropping out in the surrounding areas, thus implying a Miocene age.

\section{3.b. Gavignano}

The Gavignano-Gorga Unit (Alberti et al. 1975) is exposed in scattered outcrops around the Gavignano village (50 km SE of Rome; $41^{\circ} 42^{\prime} \mathrm{N}, 13^{\circ} 3^{\prime} \mathrm{E}$ ) and, to a lesser extent, close to the Gorga village $\left(41^{\circ} 39^{\prime} 21^{\prime \prime} \mathrm{N}\right.$, $\left.13^{\circ} 6^{\prime} 40^{\prime \prime} \mathrm{E}\right)$.

Alberti et al. (1975) recognized three lithofacies in this unit, which from base to top are as follows.

- Lower conglomeratic lithofacies. This is made of massive or crudely bedded clast-supported conglomerates with a sandy matrix and carbonate cement (Fig. 2b). The clasts are generally well rounded and poorly sorted, and display rare imbrications. They are composed of Cretaceous and Miocene limestones from the local succession with subordinate exotic Pietraforte-like sandstones. This unit unconformably overlies Upper Cretaceous carbonateplatform limestones and presents a maximum thickness of about $20 \mathrm{~m}$.

- Chaotic lithofacies. This sharply overlies the previous lithofacies and consists of very poorly sorted (from granules to boulders), angular to sub-angular clasts set in a muddy sandstone matrix with carbonate cement. The clasts are mainly from exotic lithologies: green marlstones, turbidite sandstones, 'Pietrapaesina' pelagic marly limestones, marly limestones with black and white chert, micritic limestones and subordinate igneous acidic rocks, mainly granites with pink feldspars and rhyolites with porphyritic texture. Due to its outcrop conditions and chaotic nature, the thickness of this unit cannot be precisely evaluated.

- Upper conglomeratic lithofacies. This comprises massive or thick-bedded clast-supported conglomerates with a sandy matrix and carbonate cement. The clasts are poorly sorted and consist of varied exotic lithologies: Eocene micritic limestones with planktonic foraminifera (not reported from the underlying carbonate-platform succession); reddish chert; alveolinid grainstones; and rare microgranular granites. The total thickness of this lithofacies does not exceed $20 \mathrm{~m}$.

Additionally, the three lithofacies contain scattered clasts composed of older conglomerates.

The age of these deposits is uncertain although, according to the resedimented faunas, they should be younger than Late Miocene (Alberti et al. 1975). Regarding the palaeo-environmental interpretation, the whole unit can be interpreted as alluvial-fan deposits (Nemec \& Steel, 1988; Reading, 1996). In particular, the lower and upper conglomeratic units can be related to channelized debris flows, while the chaotic unit was probably deposited from unconfined debris flows (Nemec \& Steel, 1984). This probably reflects an alternation of progradational and retrogradational fan phases, leading to the vertical stacking of proximal channelized reaches and distal lobes.

\section{3.c. Ariano Irpino}

This area presents many different terrigenous units (Fig. 3) related to the Mio-Pliocene evolution of the Central-Southern Apennines and recording the eastwards migration of the fold-and-thrust belt. As in the previous cases, the nature of the successions and the outcrop scarcity preclude precise regional correlations (Amore et al. 1988b) that are anyway beyond the purpose of the present study.

The Pliocene deposits include several terrigenous units (SGN, 1963) resting on a mainly Miocene substrate including terrigenous, carbonate and evaporitic (gypsum) deposits (Jacobacci \& Martelli, 1967). The area presents an undifferentiated assemblage of varicoloured clays of uncertain age containing Triassic and Cretaceous olistoliths and broken terrigenous and calcareous units, among which jaspers and pelagic limestones stand out.

In the first regional synthesis for these units, Ippolito et al. (1973) grouped all the terrigenous formations deposited after Middle Pliocene time in the Ariano Unit. D'Argenio, Pescatore \& Scandone (1975) recognized two main sedimentary cycles of early and middle Pliocene age. These were attributed by Amore et al. (1998) to the Ariano Group, in particular the Baronia (Zanclean-Piacenzian) and the Torrente Cervaro (middle Pliocene) formations. Each formation represents a complete shallowing and coarseningupwards cycle, from clays to sandstones and conglomerates (Ciarcia \& Torre, 1996).

Our study is focused on the Baronia Formation, in particular the Trevico Conglomerate member (BNA5 in Chiocchini, 2007; Torre, Di Nocera \& Matano, 2007), cropping out close to Ariano Irpino and Trevico villages (SGN, 1963, 2007). This unit is constituted by thick-bedded or massive, polygenic, clast-supported conglomerates showing plane-parallel lamination or gently inclined cross-bedding and occasional normal grading (Fig. 2c). Clasts are well rounded and poorly sorted, ranging from granules to pebbles with rare cobbles. They mainly consist of turbiditic sandstones and limestones from the varicoloured clays and the Mesozoic carbonate platforms (Chiocchini \& Chiocchini, 1999). Conglomerate clasts from older conglomeratic units and rare acidic igneous (granitoids) and metamorphic clasts are also present.

The age of the Trevico Conglomerates is late Zanclean - Piacenzian (Chiocchini, 2007; Torre, Di Nocera \& Matano, 2007), while the depositional setting can be related to a fluvial environment, probably dominated by gravel and subordinate sand bars (Reading, 1996).

\section{3.d. Cilento}

The Cilento region is located on the western side of the Southern Apennines and the eastern coast of 


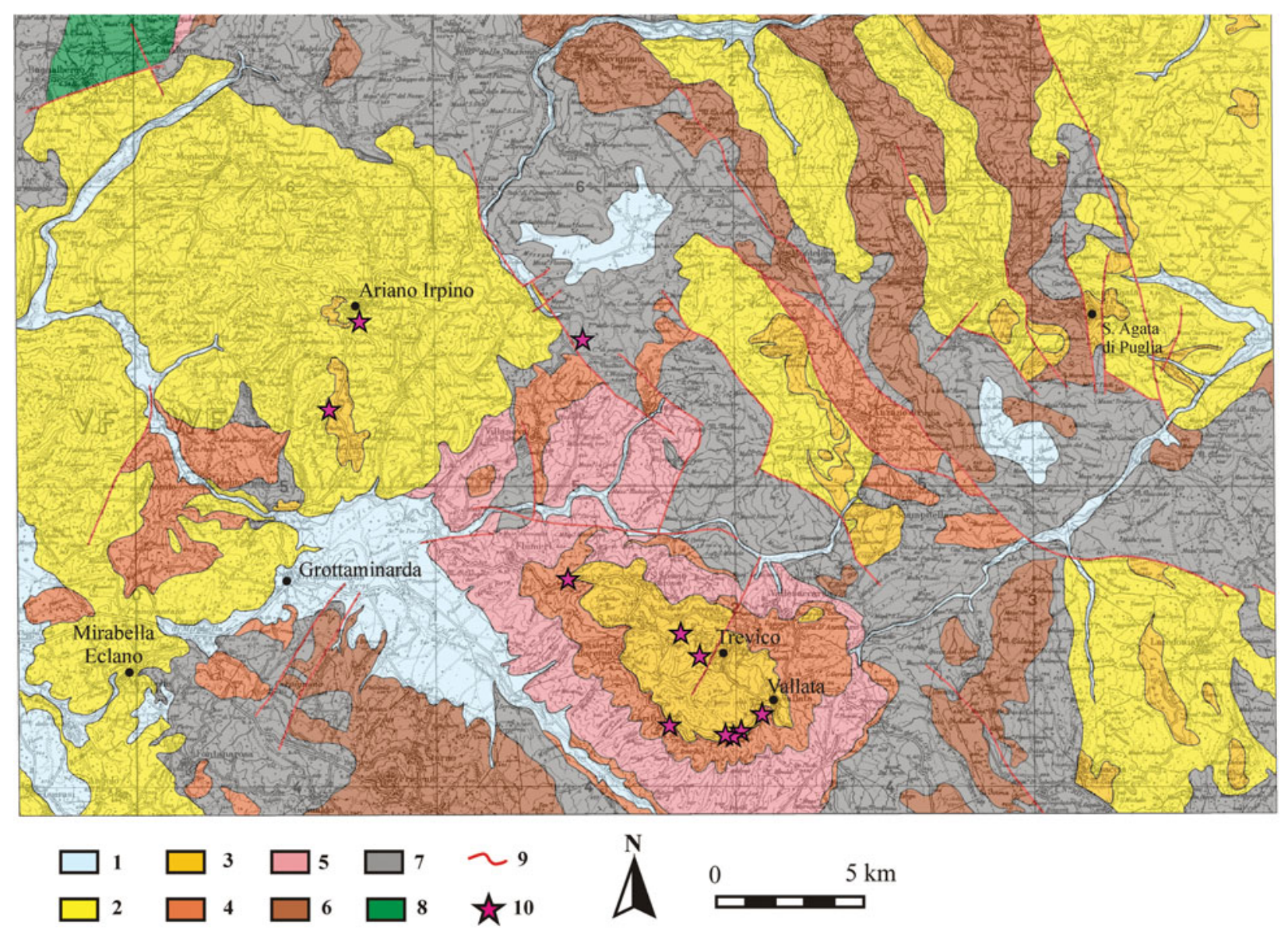

Figure 3. (Colour online) Simplified geological map of Ariano Irpino-Trevico area (modified from SGN, 1963). Key to symbols: 1, Recent alluvial deposits; 2, Pliocene sandstones and clays; 3, Pliocene conglomerates (Trevico member of the Baronia Formation); 4, Miocene sandstones; 5, Miocene clays; 6, Frigento Formation (Miocene): marls and silty clays with alternations of marly limestones and quartz-arenites; 7, varicoloured clays (uncertain age); 8, Cretaceous units; 9, main faults; and 10, location of the collected samples.

the Tyrrhenian Sea (Fig. 4). According to Martelli \& Nardi (2005), the outcropping formations can be grouped in two sets: (1) Alburno-Cervati and Monte Bulgheria carbonate units (Campano-Lucana carbonate platform); and (2) 'Internidi Units', presenting a mainly terrigenous character.

The former are constituted by Upper Triassic lower Miocene carbonate-platform deposits (Selli, 1957, 1962; Cocco, 1971; Barattolo \& Parente, 1991; Torrente et al. 2000) which tectonically overlie (Monte Bulgheria Unit, SE Cilento) and are overlain by the Internidi deposits (Alburno-Cervati Unit, NE Cilento).

The dominantly terrigenous Internidi Units were interpreted as occupying a palaeogeographic position more 'internal' (i.e. closer to the palaeo-ocean and westwards in present-day coordinates) than the Campano-Lucana carbonate platform (one of the Apenninic carbonate platforms), since they were deposited on transitional to oceanic crust (Mostardini \& Merlini, 1986; Sgrosso, 1986). Due to their sedimentary features and structural position, these units were correlated to the Liguride Complex of the Northern Apennines (Ogniben, 1969; Mostardini \& Merlini, 1986; Bonardi et al. 1988a; Bigi et al. 1992). More recently, Monaco \& Tortorici (1995) and Catalano et al. (2004) clarified the relation between the Liguride Complex and the other units cropping out in the Southern Apennines with an evolutionary model. Our study on the provenance of the exotic clasts in the CentralSouthern Apennines allows to better frame this proposed evolution within the previous Alpine collisional chain.

The terrigenous deposits of the Cilento region consist of pelagic turbidites showing a clayey-calcareous and clayey-sandy composition. They are divided into three tectonic units (e.g. Martelli \& Nardi, 2005), which are overlain by the Cilento Group (Amore et al. 1988a) and the Monte Sacro Conglomerates (Selli, 1962; De Pippo \& Valente, 1991).

The Cilento Group (previously referred to as 'Cilento Flysch'; Ietto, Pescatore \& Cocco, 1965; Cieszkowski et al. 1992, 1994) crops out all over the Cilento area, notably in the Monte Stella and Vallo della Lucania sectors. It includes the Pollica and San Mauro formations (Cocco, 1971), composed of sandy turbidites with frequent conglomerate beds. The conglomerates are generally matrix-supported, polygenic and comprise poorly sorted, moderately rounded to angular granules to cobbles formed of crystalline and metamorphic basement rocks. The matrix is composed 


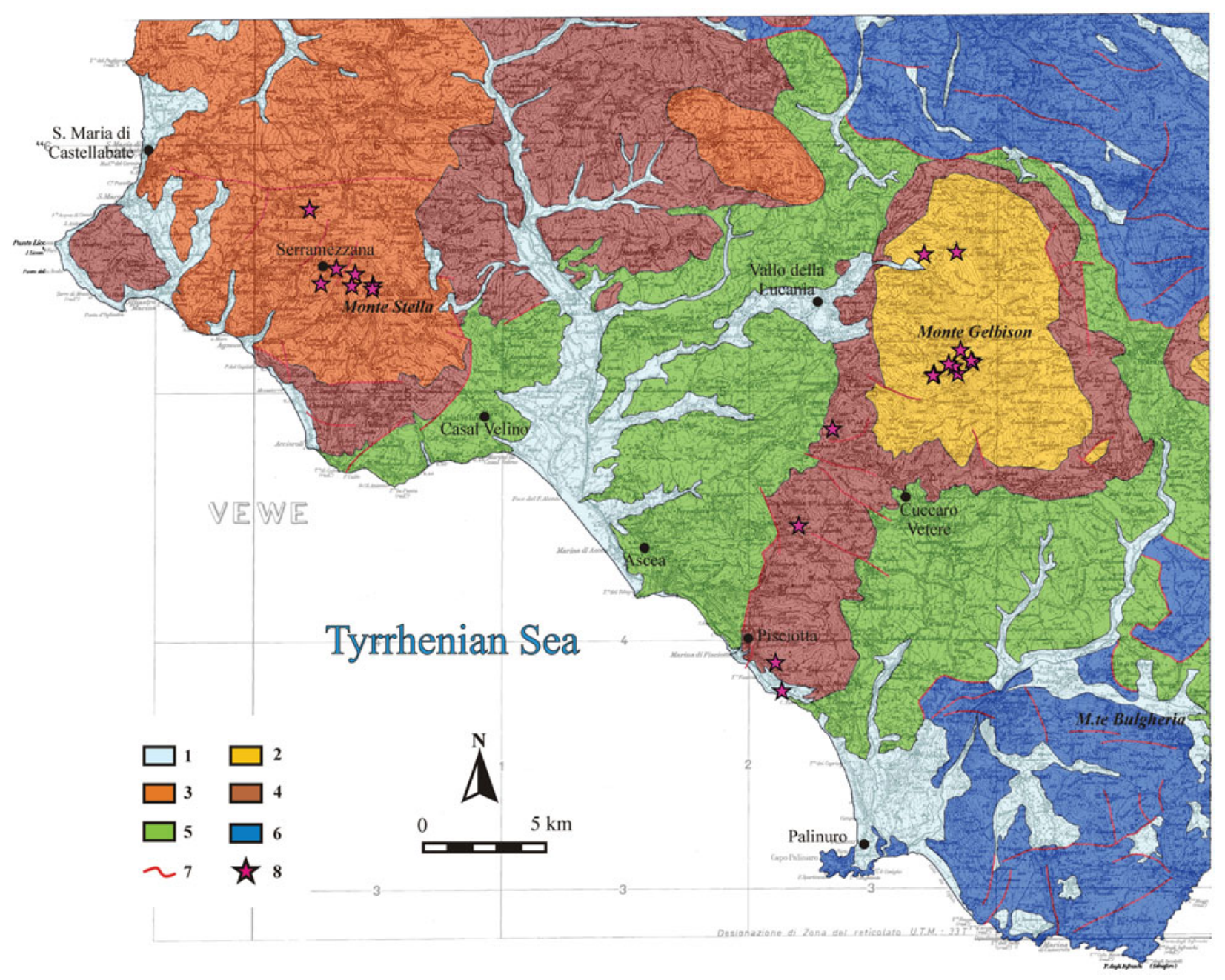

Figure 4. (Colour online) Simplified geological map of the Cilento area (modified from SGN, 1969). Key to symbols: 1, Recent alluvial deposits; 2, Monte Sacro Conglomerates (Paleocene-Oligocene); 3, San Mauro Formation (Paleocene-Oligocene); 4, Pollica Formation (Upper Cretaceous - Paleocene); 5, Ascea Formation (Cretaceous); 6, Campano-Lucana carbonate platform (Monte Bulgheria and Alburno-Cervati units, Upper Triassic - Miocene); 7, main faults; and 8, location of the collected samples.

of coarse to very coarse sand-grade particles from metamorphic and acidic igneous rocks, and subordinately from limestones and sandstones. The presence of ophiolite blocks has been referred to by Dietrich \& Scandone (1972), Di Girolamo, Morra \& Perrone (1991) and Mazzeo et al. (2016) at Monte Centaurino, east of our study area.

The Pollica and San Mauro formations have been assigned Late Cretaceous - Paleocene and PaleoceneOligocene ages, respectively (Cocco, 1971) even if, based on either literature or merely relative stratigraphic/geometric positions, younger ages have been proposed (e.g. Martelli \& Nardi, 2005). This discrepancy may easily derive from different approaches by authors in defining the depositional ages and may reflect successive reworking or tectonic stacking.

The Monte Sacro Conglomerates, with a total thickness of about $600 \mathrm{~m}$, include six lithofacies (De Pippo \& Valente, 1991) ranging from coarse conglomerates to clays. Clasts are made of sedimentary, mainly acidic, igneous and metamorphic rocks.
Conglomerates are generally matrix-supported, chaotic, massive and formed of very poorly sorted granules to boulders. Clasts are angular to moderately rounded, depending on their composition (Fig. 2d). Limestones clasts are usually less rounded than those of metamorphic and igneous rocks. The metamorphic fragments tend to have a rectangular shape due to the presence of pervasive foliation, while the igneous fragments are generally well rounded.

The conglomerates show a clear coarsening- and thickening-upwards trend, with very thick (up to $20 \mathrm{~m}$ ) massive beds composed of boulders floating in a coarse sandy matrix on top of the succession (Monte Gelbison; Fig. 2e).

According to SGN (1969), the age of the Monte Sacro Conglomerates (included in the San Mauro Formation) is Oligocene-Paleocene; Martelli \& Nardi (2005) however suggested a rather short depositional interval in early Tortonian time for this $600 \mathrm{~m}$ thick unit, based on their mere stratigraphic/geometric position. Nonetheless, this dating is still uncertain due to the nature of the deposits, the lack of biostratigraphic 
markers and the unclear relations with the underlying formations.

The general features of the Monte Sacro Conglomerates, dominated by alternating unconfined and channelized debris-flow deposits and showing a coarsening- and shallowing-upwards trend, suggest a fan-delta setting, probably passing upwards to an alluvial fan (Critelli \& Le Pera, 1990, 1994; De Pippo \& Valente, 1991; Martelli \& Nardi, 2005).

\section{Methodology}

This work is based on the study of selected outcrops from the conglomeratic units (Filettino, Gavignano, Ariano Irpino and Cilento) and laboratory analyses on selected samples. In particular, 70 stations were chosen for sampling and collection of detailed outcrop photographs, photomosaics and, when possible, general stratigraphic logs. Most of the field work was performed in the Cilento area, which exhibits the best and most continuous (both laterally and vertically) conglomerate outcrops containing exotic clasts.

The average lithologic composition of each unit was estimated from fieldwork and the analysis of 21 pictures using the graphic ImageJ software (developed at the National Institutes of Health, NIH, USA). For every picture, at least 3000 points were counted. These data were integrated with those from the literature.

With the aim of producing statistically significant sampling of the existing conglomerate population, a total of 258 samples were collected: 15 from Filettino, 16 from Gavignano, 86 from Ariano Irpino and 141 from the Cilento area. The samples were randomly taken, where possible, among the exotic clasts, focusing in particular on the igneous lithologies. Clasts of sedimentary rocks as well as the conglomerate matrix were also sampled.

A total of 78 samples was selected to make thinsections for petrographic studies: 35 of the igneous clasts and 43 of the sedimentary clasts (including the conglomerate matrix). From the total set, 23 thinsections of the intrusive lithologies and 24 of the matrix of the conglomerates and sandstone clasts were analysed by means of a point-counter stage (1000 points per section) in order to obtain the modal composition of the main constituents of the rock. For the classification, the modal compositions were plotted on a quartz - alkali feldspar - plagioclase (QAP) diagram for the intrusive rocks (Streckeisen, 1974) and on a quartz - feldspar - lithic fragments (QFL) diagram for the sandstones (Zuffa, 1980; Dickinson et al. 1983).

\section{4.a. Major- and trace-element analyses}

From the total sample set, 27 representative igneous clasts were selected for major- and trace-element analyses (including rare Earth elements or REE; Table 1). Given the nature of the samples, the size of the clast represented a significant discriminating element and only the clasts larger than $64 \mathrm{~mm}$ (cobbles and boulders) were analysed.

Major elements were analysed by x-ray fluorescence (XRF) in the Technical-Scientific Services of Oviedo University (Spain) using a WD-XRF spectrometer (model 2404, PANalytical) coupled with a $\mathrm{Rh}$ tube. Analyses were performed using glass beads of powdered rocks after fusion with lithium tetraborate. The estimated error is smaller than $1 \%$.

Trace- and rare Earth elements were analysed by inductively coupled plasma mass spectrometry (ICPMS) following sample decomposition with lithium metaborate at the Geochronology and GeochemistrySGIker facility of El País Vasco university/EHU (Spain) (for further details see García de Madinabeitia, Sánchez Lorda \& Gil Ibarguchi, 2008).

The results have been analysed and plotted using the GCDkit software, version 4.1 (Janoušek, Farrow \& Erban, 2006).

\section{Sedimentary features and petrography}

\section{5.a. Filettino}

The clasts of the Filettino conglomerates are mainly composed of grey-whitish limestones (57-85\%) and sandstones (15-43\%). The relative proportion of these main components changes through the unit, with an upwards increase of the percentage of the carbonate clasts. Sandstones are dominantly lithic-arenites with different degrees of lithification, as shown by the even or irregular (pitted) clast surfaces. Rare (usually less than $1 \%$ ) yellow micritic limestones have been found. According to Devoto (1967b), very rare igneous clasts (granitoids) are also present; nonetheless they were not found in this study.

The 15 samples were collected throughout the conglomeratic unit and comprise limestones (11 samples) and sandstones (4 samples). The former are dominantly shallow-water carbonates probably eroded from the surrounding Mesozoic carbonate formations, as also indicated by their low roundness. No igneous or metamorphic clasts were found.

The conglomerate matrix represents $21-55 \%$ of the rock, its grain size is medium to coarse sand and its composition differs from that of the clasts. It is composed of quartz (11-17\%), chert (1-4\%), feldspars $(8-12 \%)$ and lithic fragments $(34-53 \%)$; it therefore plots in the 'litharenite' field of the QFL diagram (Fig. 5a). The main components show the following features: quartz grains are well rounded and display a bimodal grain-size distribution (modes: $0.2-0.4 \mathrm{~mm}$ and $0.6-0.7 \mathrm{~mm}$ ); feldspars are generally represented by angular grains (some of which are almost idiomorphic; Fig. 6a) with a bimodal distribution (modes: $0.2 \mathrm{~mm}$ and $0.4 \mathrm{~mm}$ ); scattered coarser grains (up to $1.2 \mathrm{~mm}$ ) are present; lithic fragments are mainly of sedimentary rocks (limestones and rare sandstones) and subordinate metamorphic and igneous fragments (mainly granitoids, when recognizable). 
Table 1. Major, minor and REE composition of the analysed samples.

\begin{tabular}{|c|c|c|c|c|c|c|c|c|c|c|c|c|c|c|c|c|c|c|c|c|c|c|c|c|c|c|c|}
\hline \multirow{4}{*}{$\begin{array}{l}\text { Area } \\
\text { Rock type } \\
\text { Sample }\end{array}$} & \multicolumn{4}{|c|}{ Ariano Irpino/Trevico } & \multicolumn{23}{|c|}{ Cilento } \\
\hline & $\mathrm{g}$ & $\mathrm{g}$ & $\mathrm{g}$ & $\stackrel{\mathrm{g}}{\mathrm{P}}$ & $\mathrm{s}-\mathrm{v}$ & s-v & $\mathrm{g}$ & $\mathrm{g}$ & $\mathrm{gc}$ & $\mathrm{gc}$ & $\mathrm{gc}$ & $\mathrm{g}$ & $\mathrm{s}-\mathrm{v}$ & $\mathrm{s}-\mathrm{v}$ & $\mathrm{s}-\mathrm{v}$ & $\mathrm{g}$ & $\mathrm{gc}$ & $\mathrm{gc}$ & $\mathrm{g}$ & s-v & $\mathrm{s}-\mathrm{v}$ & $\mathrm{s}-\mathrm{v}$ & gc & $\mathrm{g}$ & $\mathrm{g}$ & $\mathrm{g}$ & $\mathrm{g}$ \\
\hline & ARI & ARI & ARI & ARI & CIL & CIL & CIL & CIL & CIL & CIL & CIL & CIL & CIL & CIL & CIL & CIL & CIL & CIL & CIL & CIL & CIL & CIL & CIL & CIL & CIL & CIL & CIL \\
\hline & $13 b$ & 03 & $13 \mathrm{a}$ & 12 & $04 a$ & $31 \mathrm{a}$ & $25 \mathrm{~b}$ & $31 \mathrm{~b}$ & $25 \mathrm{a}$ & $11 \mathrm{a}$ & $24 \mathrm{~b}$ & 07 & 23 & $04 \mathrm{c}$ & $04 \mathrm{~b}$ & 15 & 27 & 28 & $31 \mathrm{c}$ & $30 \mathrm{a}$ & $30 \mathrm{~b}$ & 16 & $24 \mathrm{c}$ & 19 & 33 & 21 & 18 \\
\hline \multicolumn{28}{|c|}{ Major elements (wt\%) } \\
\hline $\mathrm{SiO}_{2}$ & 72.33 & 75.30 & 76.50 & 80.55 & 46.97 & 62.57 & 66.91 & 67.35 & 68.88 & 69.14 & 69.36 & 69.52 & 69.64 & 69.81 & 70.84 & 72.75 & 72.88 & 72.94 & 73.21 & 73.47 & 73.53 & 73.60 & 74.92 & 75.16 & 75.79 & 76.80 & 79.74 \\
\hline $\mathrm{TiO}_{2}$ & 0.20 & 0.02 & 0.02 & 0.18 & 1.34 & 0.56 & 0.41 & 0.55 & 0.44 & 0.40 & 0.43 & 0.40 & 0.38 & 0.37 & 0.27 & 0.12 & 0.07 & 0.05 & 0.20 & 0.15 & 0.15 & 0.18 & 0.03 & 0.05 & 0.04 & 0.05 & 0.09 \\
\hline $\mathrm{Al}_{2} \mathrm{O}_{3}$ & 14.36 & 13.30 & 12.69 & 9.46 & 17.76 & 15.28 & 15.22 & 15.24 & 15.47 & 15.07 & 15.25 & 14.79 & 14.52 & 14.44 & 13.72 & 14.40 & 14.63 & 14.37 & 12.93 & 13.56 & 13.79 & 13.50 & 13.35 & 13.39 & 12.66 & 12.40 & 9.17 \\
\hline $\mathrm{Fe}_{2} \mathrm{O}_{3}$ & 1.32 & 0.51 & 0.52 & 1.73 & 7.69 & 5.10 & 3.40 & 3.84 & 3.28 & 3.10 & 3.13 & 3.08 & 3.17 & 3.13 & 2.83 & 1.32 & 0.87 & 0.78 & 2.38 & 1.82 & 1.24 & 2.26 & 0.74 & 1.21 & 0.70 & 0.66 & 0.93 \\
\hline $\mathrm{MnO}$ & 0.02 & 0.02 & 0.02 & 0.28 & 0.12 & 0.11 & 0.05 & 0.07 & 0.04 & 0.04 & 0.04 & 0.06 & 0.05 & 0.04 & 0.05 & 0.02 & 0.02 & 0.02 & 0.04 & 0.03 & 0.01 & 0.02 & 0.02 & 0.05 & 0.01 & 0.01 & 0.02 \\
\hline $\mathrm{MgO}$ & 0.38 & 0.07 & 0.09 & 0.42 & 5.85 & 3.64 & 1.79 & 1.38 & 1.12 & 1.36 & 1.12 & 1.09 & 0.97 & 0.90 & 0.98 & 0.38 & 0.37 & 0.27 & 0.51 & 0.34 & 0.30 & 0.59 & 0.20 & 0.18 & 0.16 & 0.23 & 0.39 \\
\hline $\mathrm{CaO}$ & 0.62 & 0.42 & 0.31 & 0.37 & 4.70 & 2.18 & 1.34 & 2.00 & 1.14 & 0.68 & 1.29 & 1.83 & 0.48 & 0.75 & 0.28 & 0.53 & 0.38 & 0.40 & 0.36 & 0.31 & 0.39 & 0.09 & 0.40 & 0.32 & 0.19 & 0.34 & 1.10 \\
\hline & 3.03 & 4.64 & 3.78 & 4.81 & 5.44 & 2.63 & 3.49 & 3.31 & 3.06 & 2.60 & 3.19 & 3.02 & 3.11 & 3.06 & 3.66 & 3.44 & 3.37 & 4.05 & 3.11 & 3.09 & 3.81 & 2.71 & 3.90 & 3.28 & 3.00 & 3.48 & 2.91 \\
\hline $\mathrm{K}_{2} \mathrm{O}$ & 5.37 & 4.20 & 4.93 & 0.23 & 1.08 & 3.81 & 4.29 & 3.42 & 4.20 & 4.48 & 4.04 & 4.31 & 5.04 & 4.76 & 4.57 & 4.83 & 4.95 & 4.95 & 4.95 & 5.44 & 4.93 & 4.58 & 4.80 & 4.49 & 5.76 & 4.77 & 3.05 \\
\hline $\mathrm{P}_{2} \mathrm{O}_{5}$ & 0.27 & 0.02 & 0.03 & 0.09 & 0.20 & 0.16 & 0.10 & 0.17 & 0.17 & 0.14 & 0.18 & 0.12 & 0.10 & 0.10 & 0.11 & 0.28 & 0.24 & 0.25 & 0.13 & 0.05 & 0.05 & 0.04 & 0.21 & 0.16 & 0.02 & 0.02 & 0.07 \\
\hline LOI & 1.20 & 0.64 & 0.69 & 1.07 & 8.07 & 3.63 & 2.10 & 1.85 & 1.70 & 2.69 & 1.40 & 1.18 & 1.86 & 1.88 & 1.86 & 1.35 & 1.38 & 1.07 & 1.39 & 0.98 & 0.92 & 1.97 & 0.72 & 1.09 & 0.93 & 0.81 & 1.69 \\
\hline Total & 99.10 & 99.14 & 99.58 & 99.19 & 99.22 & 99.67 & 99.10 & 99.18 & 99.50 & 99.70 & 99.43 & 99.40 & 99.32 & 99.24 & 99.17 & 99.42 & 99.16 & 99.15 & 99.21 & 99.24 & 99.12 & 99.54 & 99.29 & 99.38 & 99.26 & 99.57 & 99.16 \\
\hline \multicolumn{28}{|c|}{ Trace elements (ppm) } \\
\hline $\mathrm{Cr}$ & 2.814 & - & - & - & 135.300 & 90.850 & 14.680 & 4.866 & 2.803 & 2.624 & 3.991 & 3.390 & 2.587 & 2.991 & 1.825 & 2.249 & 1.537 & - & 1.515 & - & - & 2.379 & - & - & - & - & - \\
\hline Co & 64.78 & 71.94 & 51.96 & 61.93 & 36.97 & 22.35 & 44.75 & 52.22 & 57.92 & 81.09 & 80.95 & 51.52 & 50.45 & 63.57 & 47.70 & 99.40 & 60.76 & 12.60 & 86.38 & 15.17 & 53.57 & 45.95 & 73.66 & 216.90 & 72.39 & 61.19 & 69.44 \\
\hline $\mathrm{Ni}$ & - & - & - & - & 59.270 & 4.888 & 4.443 & - & - & - & - & - & - & - & - & - & - & - & - & - & - & - & - & - & - & - & - \\
\hline $\mathrm{Cu}$ & - & 82.510 & 60.410 & 88.040 & 66.370 & - & - & - & - & _- & _- & _- & - & 29.360 & 46.100 & - & - & - & - & - & - & - & - & - & - & - & - \\
\hline $\mathrm{Zn}$ & 54.220 & 5.305 & 9.729 & 39.560 & 109.500 & 99.810 & 51.900 & 58.580 & 60.990 & 46.560 & 60.370 & 53.980 & 66.840 & 78.740 & 43.390 & 28.230 & 21.200 & 2.120 & 43.440 & 14.400 & 12.310 & 60.900 & 10.180 & 34.770 & 12.140 & 12.170 & 16.710 \\
\hline $\mathrm{Rb}$ & 330.200 & 172.200 & 321.100 & 8.871 & 41.490 & 163.900 & 157.900 & 105.400 & 145.400 & 150.600 & 139.700 & 163.400 & 160.600 & 151.500 & 174.500 & 215.900 & 236.100 & 38.340 & 221.800 & 77.830 & 173.200 & 154.500 & 182.700 & 372.400 & 233.500 & 195.400 & 120.200 \\
\hline $\mathrm{Sr}$ & 75.43 & 21.12 & 18.01 & 23.62 & 165.30 & 264.60 & 128.50 & 197.00 & 206.20 & 136.10 & 188.50 & 139.80 & 110.40 & 107.80 & 74.03 & 63.92 & 64.01 & 16.81 & 55.97 & 25.31 & 68.71 & 69.09 & 26.71 & 29.41 & 33.83 & 28.74 & 45.18 \\
\hline
\end{tabular}


Table 1. Continued.

\begin{tabular}{|c|c|c|c|c|c|c|c|c|c|c|c|c|c|c|c|c|c|c|c|c|c|c|c|c|c|c|c|}
\hline \multirow{4}{*}{$\begin{array}{l}\text { Area } \\
\text { Rock type } \\
\text { Sample }\end{array}$} & \multicolumn{4}{|c|}{ Ariano Irpino/Trevico } & \multicolumn{23}{|c|}{ Cilento } \\
\hline & $\mathrm{g}$ & $\mathrm{g}$ & $\mathrm{g}$ & $\mathrm{g}$ & $\mathrm{s}-\mathrm{v}$ & $\mathrm{s}-\mathrm{v}$ & $\mathrm{g}$ & $\mathrm{g}$ & $\mathrm{gc}$ & $\mathrm{gc}$ & $\mathrm{gc}$ & $\mathrm{g}$ & $\mathrm{s}-\mathrm{v}$ & $\mathrm{s}-\mathrm{v}$ & $\mathrm{s}-\mathrm{v}$ & $\mathrm{g}$ & $\mathrm{gc}$ & $\mathrm{gc}$ & $\mathrm{g}$ & $\mathrm{s}-\mathrm{v}$ & s-v & $\mathrm{s}-\mathrm{v}$ & $\mathrm{gc}$ & $\mathrm{g}$ & $\mathrm{g}$ & $\mathrm{g}$ & $\mathrm{g}$ \\
\hline & ARI & ARI & ARI & ARI & CIL & CIL & CIL & $\mathrm{CIL}$ & CIL & CIL & $\mathrm{CIL}$ & CIL & $\mathrm{CIL}$ & $\mathrm{CIL}$ & $\mathrm{CIL}$ & $\mathrm{CIL}$ & $\mathrm{CIL}$ & $\mathrm{CIL}$ & CIL & CIL & $\mathrm{CIL}$ & CIL & $\mathrm{CIL}$ & $\mathrm{CIL}$ & $\mathrm{CIL}$ & $\mathrm{CIL}$ & CIL \\
\hline & $13 \mathrm{~b}$ & 03 & $13 a$ & 12 & $04 \mathrm{a}$ & $31 \mathrm{a}$ & $25 \mathrm{~b}$ & $31 \mathrm{~b}$ & $25 \mathrm{a}$ & $11 \mathrm{a}$ & $24 \mathrm{~b}$ & 07 & 23 & $04 \mathrm{c}$ & $04 \mathrm{~b}$ & 15 & 27 & 28 & $31 \mathrm{c}$ & $30 \mathrm{a}$ & $30 \mathrm{~b}$ & 16 & $24 \mathrm{c}$ & 19 & 33 & 21 & 18 \\
\hline $\mathrm{Y}$ & 7.678 & 46.800 & 31.660 & 17.340 & 47.090 & $\begin{array}{l}0 \\
0\end{array} 23.800$ & 17.570 & 26.870 & 23.330 & 19.470 & $\begin{array}{ll}0 & 19.300\end{array}$ & 34.180 & 32.720 & 33.480 & $\begin{array}{l}0 \quad 20.750 \\
\end{array}$ & 10.530 & 14.790 & 1.233 & 29.220 & 10.060 & 54.980 & 30.910 & 11.270 & 20.350 & 24.310 & 023.200 & 8.550 \\
\hline $\mathrm{Zr}$ & 89.460 & 39.340 & 43.020 & 60.540 & 209.000 & 0171.400 & 111.600 & 190.400 & 206.800 & 193.600 & 0186.800 & 137.900 & 199.800 & 205.000 & 0143.200 & 57.580 & 35.420 & 4.578 & 137.300 & 64.910 & 303.000 & 144.400 & 28.950 & 31.340 & 56.630 & 045.210 & 47.920 \\
\hline $\mathrm{Nb}$ & 12.650 & 6.257 & 7.542 & 13.250 & 9.190 & $\begin{array}{l}0 \\
0\end{array}$ & 7.617 & 15.950 & 18.260 & 16.970 & $\begin{array}{ll}0 & 17.900\end{array}$ & 13.850 & 16.110 & 17.270 & $\begin{array}{ll}0 & 11.150\end{array}$ & 10.230 & 13.490 & - & 15.710 & 4.007 & 20.430 & 14.050 & 4.065 & 16.470 & 5.650 & $\begin{array}{ll}0 & 4.592\end{array}$ & $2 \quad 5.375$ \\
\hline Sn & 10.040 & 1.627 & 3.164 & 7.016 & 1.805 & $\begin{array}{ll}5 & 12.430\end{array}$ & 3.288 & 3.209 & 1.892 & 1.869 & $\begin{array}{ll}9 & 2.411\end{array}$ & 3.714 & 1.951 & 2.063 & $\begin{array}{l}3 \quad 2.511\end{array}$ & 8.519 & 19.310 & 1.143 & 12.320 & 0.977 & 3.284 & 4.349 & 9. 3.454 & 10.560 & 3.356 & 2.307 & 2.600 \\
\hline Cs & 7.088 & 1.930 & 4.719 & 0.819 & 1.552 & $2 \quad 4.964$ & $\quad 3.938$ & 2.361 & 4.141 & 2.537 & $\begin{array}{ll}7 & 3.579\end{array}$ & 5.177 & 2.225 & 2.675 & $\begin{array}{ll}5 & 4.817\end{array}$ & 5.838 & 14.190 & 1.706 & 3.735 & 1.456 & 4.853 & 2.477 & $7 \quad 5.613$ & 9.218 & $\begin{array}{l}3.962 \\
\end{array}$ & $\begin{array}{ll}2 & 2.840\end{array}$ & $\quad 2.180$ \\
\hline $\mathrm{Ba}$ & 302.50 & 59.46 & 128.10 & 43.18 & 431.90 & 985.70 & 496.10 & 842.40 & 1200.00 & 974.90 & 965.40 & 670.70 & 1077.00 & 838.60 & 525.10 & 321.00 & 284.60 & 40.72 & 474.40 & 160.70 & 939.80 & 865.60 & 60.62 & 167.70 & 80.36 & 90.13 & 278.40 \\
\hline $\mathrm{Hf}$ & 2.638 & 2.278 & 2.746 & 2.117 & 4.591 & 14.431 & 3.099 & 5.084 & 5.489 & 4.971 & $1 \quad 5.006$ & 4.156 & 5.457 & 5.688 & $8 \quad 4.092$ & 1.815 & 1.259 & 0.181 & 4.465 & 1.988 & 8.347 & 4.549 & 1.448 & 1.394 & $\begin{array}{l}4 \\
4.558\end{array}$ & $\begin{array}{ll}8 & 2.189\end{array}$ & $9 \quad 1.615$ \\
\hline $\mathrm{Ta}$ & 1.910 & 2.630 & 4.425 & 2.337 & 0.412 & 0.863 & 0.948 & 1.134 & 1.308 & 1.106 & 1.383 & 1.630 & 1.112 & 1.261 & 1.089 & 2.164 & 3.823 & - & 2.649 & 0.205 & 1.583 & 1.349 & 1.066 & 3.554 & $\begin{array}{l}4 \\
4.394\end{array}$ & 1.711 & 1.361 \\
\hline $\mathrm{Pb}$ & 33.020 & 23.660 & 22.720 & 2.008 & 8.111 & $1 \quad 23.070$ & 16.450 & 19.490 & 22.660 & 18.790 & $\begin{array}{ll}0 & 19.280\end{array}$ & 29.640 & 54.440 & 68.630 & $\begin{array}{ll}0 & 14.020\end{array}$ & 30.520 & 26.070 & 5.288 & 31.750 & 9.432 & 20.750 & 6.316 & 643.450 & 16.240 & 33.230 & 027.640 & 9.654 \\
\hline Th & 13.740 & 11.850 & 16.590 & 11.660 & 6.698 & 13.370 & 11.710 & 14.270 & 15.700 & 12.900 & $\begin{array}{ll}0 & 13.310\end{array}$ & 15.700 & 15.280 & 15.200 & $\begin{array}{ll}0 & 10.430\end{array}$ & 6.257 & 2.617 & 0.823 & 17.350 & 7.919 & 20.430 & 16.590 & 7.346 & 4.976 & 516.010 & 022.660 & 8.537 \\
\hline U & 3.597 & 2.156 & 1.513 & 0.915 & 1.618 & 4.160 & 1.554 & 2.513 & 1.574 & 1.670 & $\begin{array}{ll}0 & 1.516\end{array}$ & 2.605 & 3.828 & 4.338 & $\begin{array}{l}8 \quad 3.209\end{array}$ & 2.118 & 1.721 & 0.006 & 4.127 & 1.106 & 4.561 & 2.510 & 2.350 & 1.993 & $\begin{array}{l}3.474 \\
\end{array}$ & $\begin{array}{ll}4 & 2.080\end{array}$ & 3.328 \\
\hline $\mathrm{La}$ & 21.350 & 14.410 & 2.797 & 17.420 & 24.590 & $\begin{array}{ll}0 & 33.890\end{array}$ & 25.480 & 38.410 & 49.200 & 46.350 & $\begin{array}{ll}0 & 42.140\end{array}$ & 32.400 & 38.880 & 36.290 & $\begin{array}{ll}0 & 21.630\end{array}$ & 11.900 & 10.870 & 0.549 & 21.230 & 17.220 & 61.350 & 43.770 & 2.683 & 12.860 & 13.260 & $\begin{array}{ll}0 & 6.521\end{array}$ & 17.960 \\
\hline $\mathrm{Ce}$ & 51.180 & 29.880 & 12.660 & 36.280 & 40.630 & $\begin{array}{ll}0 & 69.870\end{array}$ & 47.380 & 82.830 & 101.900 & 93.780 & $\begin{array}{ll}0 & 88.530\end{array}$ & 68.440 & 85.940 & 84.790 & $\begin{array}{ll}0 & 39.780\end{array}$ & 25.130 & 14.860 & 0.430 & 49.240 & 39.380 & 131.200 & 92.190 & 6.241 & 25.210 & 28.350 & $\begin{array}{lll}0 & 15.150\end{array}$ & 39.660 \\
\hline $\operatorname{Pr}$ & 6.219 & 3.913 & 0.753 & 4.462 & 26.514 & 8.315 & 5.533 & 9.919 & 12.310 & 11.410 & $\begin{array}{ll}0 & 10.490\end{array}$ & 8.275 & 10.020 & 9.624 & $\begin{array}{l}4 \quad 5.187\end{array}$ & 2.898 & 2.659 & - & 5.476 & 4.127 & 14.790 & 11.050 & 0.656 & 3.350 & 3.462 & $2 \quad 2.013$ & $\begin{array}{l}3 \\
3\end{array} 4.419$ \\
\hline $\mathrm{Nd}$ & 23.070 & 14.420 & 3.487 & 16.740 & 26.680 & $\begin{array}{ll}0 & 30.540\end{array}$ & 19.250 & 37.410 & 46.090 & 41.480 & $\begin{array}{ll}0 & 39.020\end{array}$ & 31.360 & 37.130 & 35.820 & $\begin{array}{ll}0 & 19.050\end{array}$ & 10.180 & 10.000 & - & 19.990 & 15.010 & 54.890 & 39.930 & 2.579 & 12.270 & 12.770 & $\begin{array}{ll}0 & 8.093\end{array}$ & 14.550 \\
\hline $\mathrm{Sm}$ & 4.978 & 4.519 & 1.950 & 3.873 & 5.696 & $\begin{array}{l}6 \\
6.525\end{array}$ & 3.368 & 7.098 & 8.298 & 7.095 & $5 \quad 7.043$ & 6.375 & 6.938 & 6.959 & $\begin{array}{l}9 \\
9.798\end{array}$ & 2.252 & 2.321 & 0.078 & 4.356 & 2.685 & 10.060 & 7.396 & $\begin{array}{ll}5 & 0.905\end{array}$ & 3.071 & 2.983 & 2.481 & 2.276 \\
\hline $\mathrm{Eu}$ & 0.482 & 0.125 & 0.070 & 0.239 & 1.204 & 1.033 & 0.832 & 1.168 & 1.305 & 1.072 & 1.209 & 0.976 & 1.012 & 1.043 & 0.627 & 0.512 & 0.576 & 0.063 & 0.392 & 0.203 & 1.413 & 0.804 & $4 \quad 0.106$ & 0.344 & $\begin{array}{l}4 \\
0.237\end{array}$ & $\begin{array}{ll}7 & 0.228\end{array}$ & 0.325 \\
\hline $\mathrm{Gd}$ & 3.266 & 4.932 & 2.687 & 3.398 & 5.612 & 4.359 & 2.934 & 5.472 & 6.149 & 5.216 & 5.168 & 5.323 & 5.505 & 5.581 & 3.363 & 2.087 & 2.194 & 0.317 & 3.906 & 2.198 & 8.182 & 5.741 & 1.153 & 2.810 & 2.723 & 2.515 & 1.702 \\
\hline $\mathrm{Tb}$ & 0.370 & 1.004 & 0.612 & 0.556 & & 0.643 & & 0.791 & & 0.675 & & 0.842 & & 0.864 & 0.535 & 0.337 & 0380 & 0.048 & 0.663 & 0.306 & 1.289 & 0.835 & 0.227 & 0.516 & $\begin{array}{ll}5 & 0.490\end{array}$ & 0.470 & 0.236 \\
\hline Dy & 1.589 & 6.397 & 4.300 & 3.233 & 5.711 & 3.676 & 2.587 & 4.291 & 4.060 & 3.388 & 3.409 & 4.986 & 4.797 & 5.068 & 3.264 & 1.868 & 2.281 & 0.380 & 4.190 & 1.778 & 7.726 & 4.758 & $\begin{array}{l}3.590 \\
\end{array}$ & 3.147 & 7.225 & 3.134 & 1.385 \\
\hline Ho & 0.158 & 0.951 & 0.677 & 0.426 & 0.898 & 0.514 & 0.362 & 0.583 & & 0.437 & & 0.724 & 0.690 & 0.737 & 0.488 & 0.215 & 0.291 & 0.032 & 0.625 & 0.235 & 1.185 & 0.682 & 20.221 & 0.429 & 0.501 & 0.472 & 0.186 \\
\hline $\mathrm{Er}$ & 0.592 & 3.891 & 2.883 & 1.739 & 3.588 & 2.070 & 1.524 & 2.243 & 1.914 & 1.644 & 1.588 & 2.938 & 2.758 & 2.970 & 2.088 & 0.801 & 1.106 & 0.176 & 2.674 & 1.000 & 4.908 & 2.750 & 0.993 & 1.743 & $\begin{array}{l}3 \\
2.193\end{array}$ & 2.035 & 0.813 \\
\hline $\mathrm{Tm}$ & 0.081 & 0.643 & 0.492 & 0.279 & 0.545 & 0.328 & 0.244 & 0.349 & 0.282 & 0.242 & 0.233 & 0.487 & 0.436 & 0.474 & 0.349 & 0.113 & 0.162 & 0.021 & 0.456 & 0.152 & 0.804 & 0.442 & 20.173 & 0.292 & 20.382 & 0.351 & 0.133 \\
\hline $\mathrm{Yb}$ & 0.559 & 3.888 & 3.193 & 1.859 & 3.258 & 2.109 & 1.655 & 2.202 & 1.709 & 1.496 & 1.459 & 3.175 & 2.768 & 2.980 & 2.287 & 0.718 & 1.015 & 0.199 & 3.038 & 1.017 & 5.030 & 2.760 & 1.280 & 1.911 & 2.586 & 2.279 & 0.963 \\
\hline $\mathrm{Lu}$ & 0.063 & 0.620 & 0.517 & 0.285 & 0.535 & 0.335 & 0.260 & 0.347 & 0.257 & 0.224 & 0.217 & 0.500 & 0.440 & 0.471 & 0.373 & 0.089 & 0.138 & 0.008 & 0.503 & 0.151 & 0.846 & 0.432 & 20.207 & 0.284 & $4 \quad 0.433$ & $\begin{array}{ll}3 & 0.374\end{array}$ & 0.146 \\
\hline
\end{tabular}

$\mathrm{g}$ - granitoid; gc - cordierite-bearing granite; s-v - subvolcanic. 

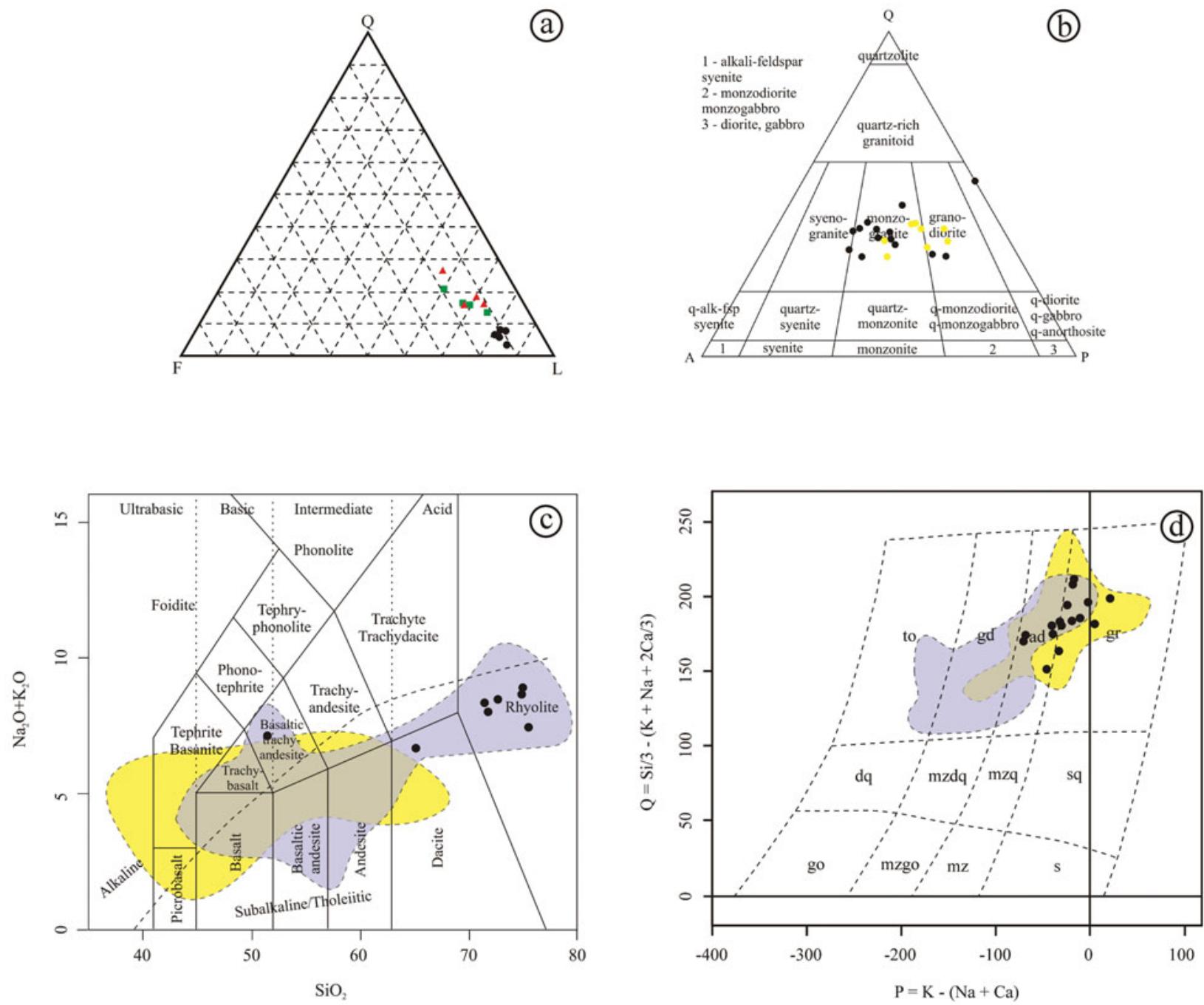

Figure 5. (Colour online) (a) QFL diagram of the arenaceous matrix of the conglomerates. Black dots: Cilento samples; red triangles: Filettino samples; green squares: Gavignano samples. (b) QAP diagram of the intrusive samples (Streckeisen, 1974). Yellow dots indicate cordierite-bearing granitoids. (c) TAS diagram (Le Bas et al. 1986) of the subvolcanic samples; (d) P-Q diagram (Debon \& LeFort, 1983) of the intrusive samples. The shaded areas indicate the field of existence of rocks from the two possible source areas. Yellow: Calabria; violet: Sardinia (data taken from the literature, see text).

\section{5.b. Gavignano}

The composition of the whole unit is dominated by calcareous fragments (90-94\%) and subordinate sandstones $(6-10 \%)$. In contrast to the Filettino conglomerates, no clear trends in clast composition are present.

The 16 collected samples come from the 3 different lithofacies and are composed of sedimentary rocks, limestones (5 samples) and sandstones (11 samples). No igneous or metamorphic rocks were found. The limestones are dominantly shallow-water carbonates, some with gastropods, molluscs and rare benthic foraminifera (Fig. 6b).

The matrix is very similar to that of the Filettino conglomerates. It represents $40-51 \%$ of the rock and its grain size varies between medium and coarse sand. The most striking feature of the matrix is its different composition with respect to clasts. It is made of quartz (11-16\%), chert (0-2\%), feldspars (9-15\%) and igneous and metamorphic rock fragments (45-
$64 \%)$. It can therefore be classified as 'litharenite' in the QFL diagram (Fig. 5a). The main characteristics of the grains are: quartz grains are usually well rounded, with a bimodal grain size distribution (modes: 0.3$0.4 \mathrm{~mm}$ and $0.6-0.7 \mathrm{~mm}$ ); feldspar grains are angular, nearly intact crystals, some of which are almost idiomorphic (Fig. 6b) and also show a bimodal size distribution $(0.2 \mathrm{~mm}$ and $0.4-0.6 \mathrm{~mm})$, although coarser grains (up to $1.5 \mathrm{~mm}$ ) are present; lithic fragments are mainly of sedimentary rocks (33-57\% of the matrix), in particular of limestones with subordinate sandstones; and the rest is made up of metamorphic (3.5\%$10 \%$ of the matrix) and igneous ( $8-20 \%$ of the matrix) fragments (the latter are dominantly granitoids when recognizable).

\section{5.c. Ariano Irpino}

The 86 collected samples come from different stratigraphic levels of the Baronia Formation, in 


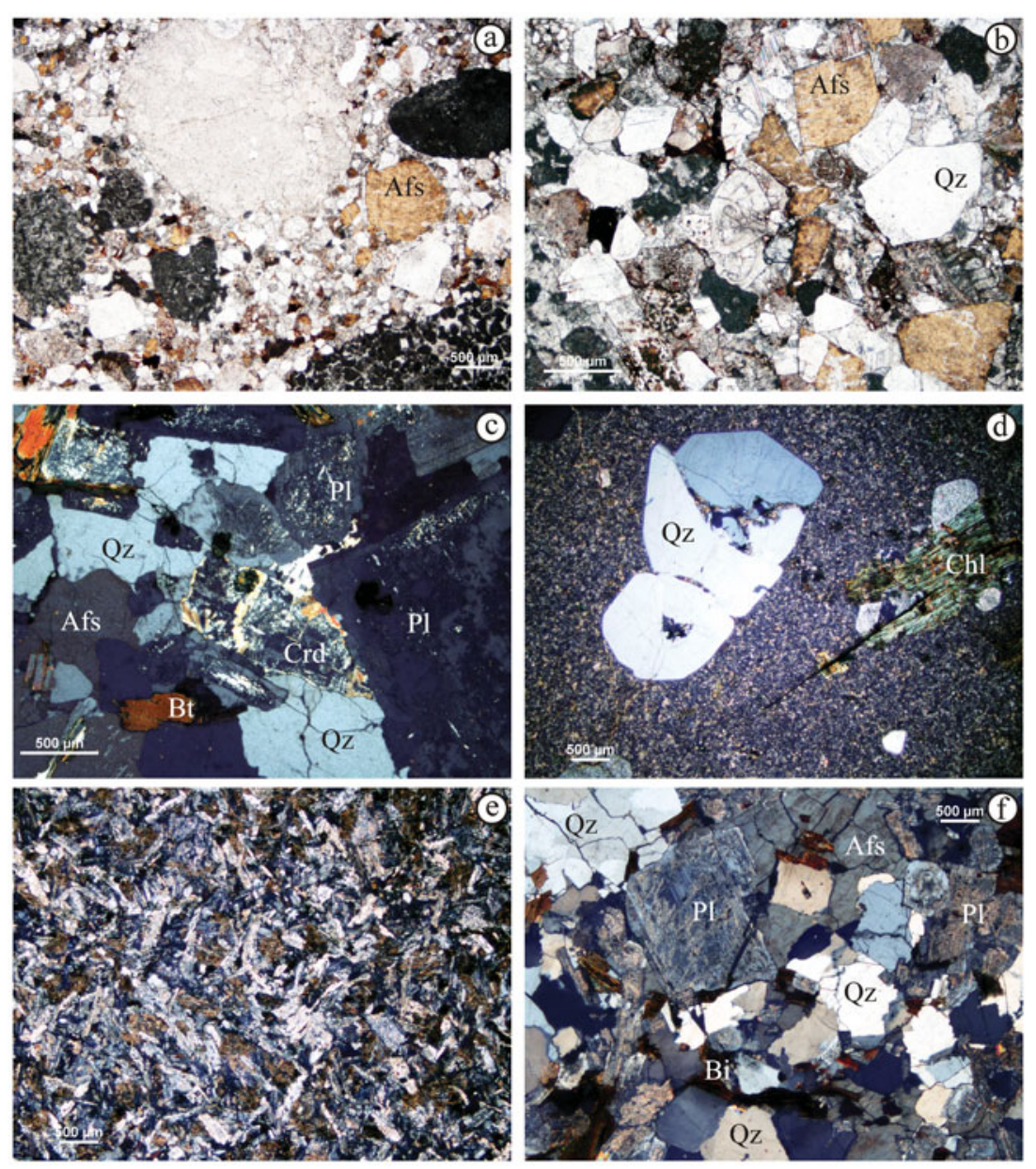

Figure 6. (Colour online) Photographs of representative thin-sections from the studied conglomeratic units. (a) Filettino conglomerates, sample FIL01c. Note the presence of feldspars, quartz and peloidal/bioclastic limestones (black fragments). (b) Gavignano conglomerates, sample VOL01a. Note the presence of feldspars, quartz, shallow-water limestones and large benthic foraminifera (centre of the image). (c) Cordierite-bearing granite, sample CIL25a; crossed Nicols. (d) Porphyritic rhyodacite, sample CIL16, crossed Nicols. (e) Subvolcanic basaltic trachy-andesite, sample CIL04a, crossed Nicols. The texture is given by the plagioclase crystals. (f) Monzogranite, sample CIL11a, crossed nichols. Mineral abbreviations (Whitney \& Evans, 2010). Afs - alkali feldspar; Qz - quartz; Pl plagioclase; $\mathrm{Bt}$ - biotite; $\mathrm{Crd}$ - cordierite; $\mathrm{Chl}$ - chlorite.

particular the Trevico Conglomerates member (BNA5 of Chiocchini, 2007 and Torre, Di Nocera \& Matano, 2007) (Fig. 3). They are represented by sedimentary (65 samples, of which 14 limestones, 43 sandstones and 8 cherts/jaspers) and igneous (21 samples) rocks. The latter generally show a high degree of alteration, which varies with the average clast size and rounding. In particular, the coarser clasts are usually less rounded and weathered than the finer ones. The igneous samples are entirely represented by acidic intrusive lithotypes (granitoids), in particular monzogranites and tonalites according to the modal composition (Fig. 5b, d; Table 2).

The matrix is scarce (less than 10\%) and even locally absent, resulting in an openwork conglomerate. It comprises reddish medium- to coarse-grade sand, composed of quartz, chert, feldspars and lithic fragments. The latter are mainly of sedimentary rocks (sandstones and subordinate limestones) with minor metamorphic and acidic igneous fragments. Given the scarcity of the matrix, no detailed compositional studies have been performed.

\section{5.d. Cilento}

The collected samples come from the Cilento Group (Pollica and San Mauro formations) and the Monte Sacro Conglomerates. The conglomerate clasts are composed of sedimentary, metamorphic and igneous rocks. Their relative proportion varies widely among the studied outcrops.

The number of clasts of sedimentary rocks varies from $0 \%$ to $77 \%$ and they range from granules to cobbles. They dominantly comprise grey limestones and subordinately sandstones. Limestone clasts in some cases contain shallow-water fauna (gastropods and benthic foraminifera). These have undergone selective dissolution leading to moldic pores, in some cases with remnants of the dissolved clast.

Clasts of metamorphic rocks (representing 2-52\% in the studied outcrops) are mainly represented by phyllites, schists, rare gneisses and quartzites as well as by grains of metamorphic quartz. Subordinate whitishyellow marble clasts have also been found. Clast size ranges from granules to boulders $40-50 \mathrm{~cm}$ in 
Table 2. Modal composition of selected igneous samples.

\begin{tabular}{|c|c|c|c|c|c|c|c|c|c|c|}
\hline Sample & Rock type & Qz & $\mathrm{Kfs}$ & Pl & $\mathrm{Bt}$ & Ms & Crd & Chl & Total & Observations \\
\hline ARI03 & Monzogranite & 357 & 307 & 320 & 11 & 5 & 0 & 0 & 1000 & \\
\hline ARI12 & Tonalite & 509 & 0 & 433 & 58 & 0 & 0 & 0 & 1000 & \\
\hline ARI13a & Monzogranite & 339 & 307 & 339 & 15 & 0 & 0 & 0 & 1000 & \\
\hline ARI13b & Monzogranite & 349 & 303 & 243 & 31 & 71 & 0 & 3 & 1000 & \\
\hline CIL05 & Granodiorite & 310 & 143 & 420 & 63 & 15 & 23 & 26 & 1000 & Cordierite-bearing \\
\hline CIL07a & Monzogranite & 298 & 406 & 270 & 12 & 1 & 0 & 13 & 1000 & Episyenite \\
\hline CIL07b & Monzogranite & 382 & 368 & 219 & 25 & 3 & 0 & 3 & 1000 & \\
\hline CIL07d & Monzogranite & 347 & 278 & 283 & 68 & 0 & 0 & 24 & 1000 & \\
\hline CIL11 & Granodiorite & 358 & 141 & 413 & 30 & 7 & 4 & 47 & 1000 & Cordierite-bearing \\
\hline CIL11a & Monzogranite & 355 & 206 & 312 & 76 & 6 & 19 & 26 & 1000 & Cordierite-bearing \\
\hline CIL15 & Monzogranite & 326 & 308 & 258 & 16 & 78 & 0 & 14 & 1000 & \\
\hline CIL19 & Monzogranite & 444 & 221 & 291 & 14 & 30 & 0 & 0 & 1000 & \\
\hline CIL21 & Monzogranite & 409 & 346 & 236 & 3 & 0 & 0 & 6 & 1000 & \\
\hline CIL24b & Monzogranite & 349 & 192 & 347 & 71 & 13 & 20 & 8 & 1000 & Cordierite-bearing \\
\hline CIL24c & Monzogranite & 322 & 221 & 419 & 0 & 13 & 16 & 9 & 1000 & Cordierite-bearing \\
\hline CIL25a & Monzogranite & 350 & 191 & 316 & 80 & 18 & 32 & 13 & 1000 & Cordierite-bearing \\
\hline CIL25b & Granodiorite & 287 & 206 & 419 & 0 & 0 & 0 & 88 & 1000 & Episyenite \\
\hline CIL27 & Monzogranite & 327 & 309 & 290 & 6 & 27 & 29 & 12 & 1000 & Cordierite-bearing \\
\hline CIL28 & Monzogranite & 293 & 337 & 333 & 1 & 12 & 14 & 10 & 1000 & Cordierite-bearing \\
\hline CIL31b & Granodiorite & 275 & 172 & 441 & 44 & 0 & 0 & 68 & 1000 & \\
\hline CIL31c & Syenogranite & 306 & 412 & 213 & 58 & 1 & 0 & 10 & 1000 & Episyenite \\
\hline CIL33 & Syenogranite & 384 & 399 & 210 & 6 & 0 & 0 & 1 & 1000 & \\
\hline
\end{tabular}

diameter and they show different degrees of rounding. Foliated lithologies (phyllites, schists) tend to yield more angular clasts than non-foliated quartzites and marbles).

Clasts of igneous rocks (representing $17-70 \%$ in the studied outcrops) are composed almost exclusively of acidic rocks, both intrusive (granitoids) and subvolcanic with a porphyritic texture (rhyolites and dacites). Clasts are usually well rounded and vary from granules to boulders $40-50 \mathrm{~cm}$ in size.

This composition is in agreement with data from Critelli \& Le Pera (1990) and Critelli (1991).

There is an upwards increase in the abundance of igneous and metamorphic versus sedimentary clasts. This is in contrast with De Pippo \& Valente (1991), who reported an opposite trend. This compositional variation is accompanied by a coarsening-upwards trend, from an average clast size of about $3 \mathrm{~cm}$ at the base to $12-15 \mathrm{~cm}$ in the uppermost beds. The igneous clasts show a larger upwards increase of the average size with respect to the others, while the metamorphic clasts show no clear variations.

The 141 samples have been collected from several stratigraphic layers of the San Mauro and Monte Sacro Conglomerates formations. They comprise sedimentary (12 samples), igneous (106 samples) and metamorphic (3 samples) rocks. Finally, the matrix of the conglomerate has been studied in 18 samples. The sedimentary clasts are composed of sandstones (10 samples) and limestones (2 samples), while the igneous clasts are represented by dominantly acidic rocks which can be grouped into two subsets: intrusive (87 samples) and subvolcanic porphyritic rocks (19 samples).

Intrusive lithologies are represented by syenogranites, monzogranites and granodiorites according to their modal composition (Figs 5b, 6f; Table 2) and the P-Q diagram of Debon \& LeFort (1983; Fig. 5d). It is important to note the abundance of cordierite-bearing granites ( 8 out of 18 analysed samples: CIL05, CIL11, CIL11a, CIL24b, CIL24c, CIL25a, CIL27 and CIL28; Fig. 6c).

Three samples (CIL07a, CIL25b, CIL31c) show intense hydrothermal alteration as a result of fluid-rock interaction after solidification. In particular, the rocks were subjected to silica depletion with the formation of episyenites (Cathelineau, 1986; Recio et al. 1997).

Subvolcanic lithologies are represented by porphyritic rocks, mainly rhyolites and dacites according to the total alkali versus silica (TAS) diagram of Le Bas et al. (1986) (Figs 5c, 6d). Only one of the analysed samples is composed of a basaltic trachyandesite (CIL04a, Figs 5c, 6e).

The matrix of the conglomerate is very abundant, usually representing $55-86 \%$ of the rock. It is dominantly composed of lithic fragments (45-85\%, mainly metamorphic and igneous acidic rock fragments), with subordinate quartz grains (usually less than $10 \%$ ), feldspars (10-15\%) and micas (usually less than $2 \%$ ).

\section{Geochemistry}

The geochemical analysis of the igneous clasts of the studied Tertiary conglomeratic units from the CentralSouthern Apennines represents a good test to establish the genetic relationship among the samples collected in the four localities and the known igneous complexes of Sardinia and Calabria. These have been identified as the most probable source areas due to the presence of crystalline and metamorphic basement rocks, and their palaeo-position before the opening of the Tyrrhenian Sea.

We compared the major- and minor-element (including REE) composition of the selected clasts and the igneous rocks of the Palaeozoic basement of Sardinia and Calabria. A similar methodology has 

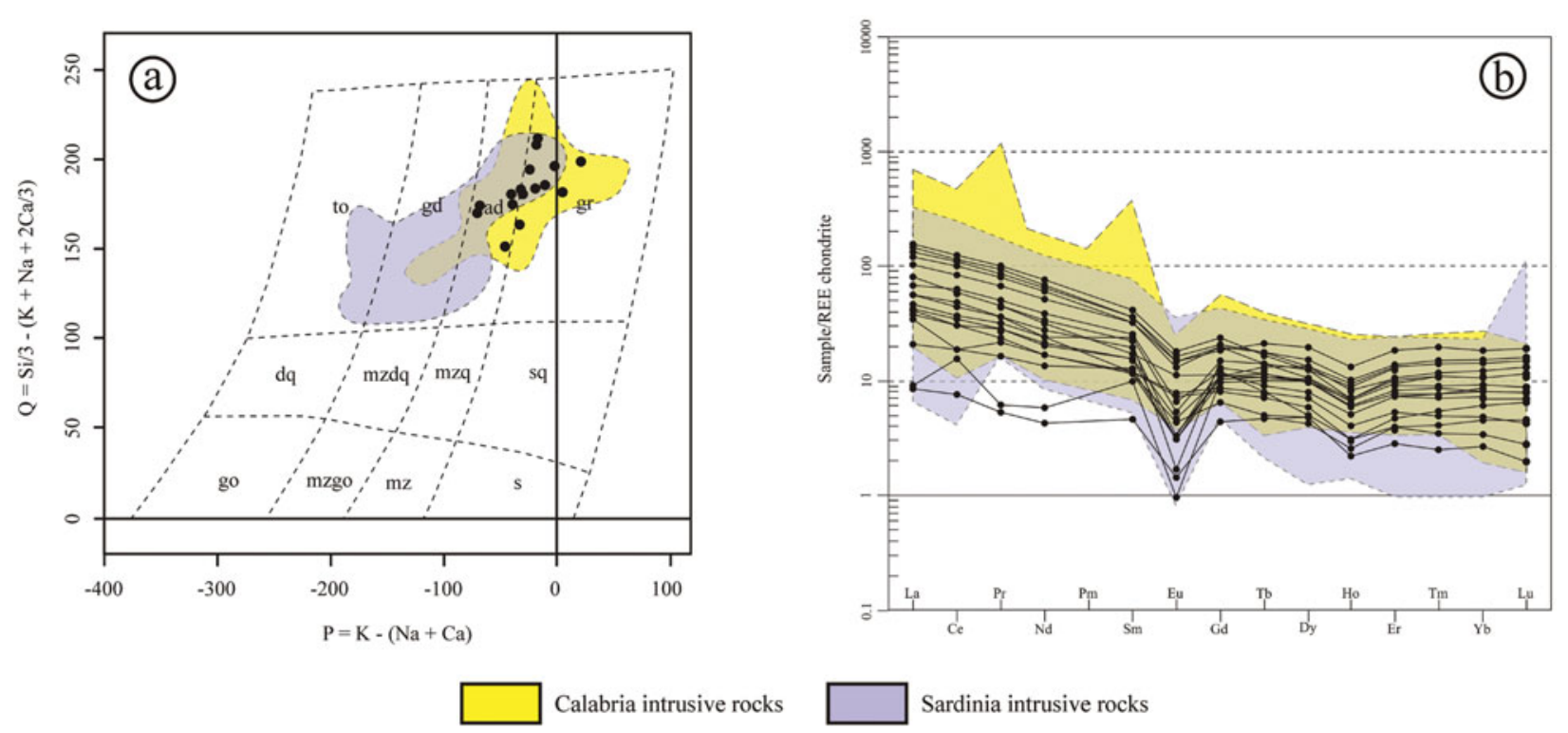

Figure 7. (Colour online) Geochemical features of the analysed intrusive samples: (a) P-Q diagram (Debon \& LeFort, 1983); and (b) REE normalized patterns (Boynton, 1984). The shaded areas indicate the field of existence of intrusive rocks from the two possible source areas. Yellow: Calabria; violet: Sardinia (data taken from the literature, see text).

been successfully applied to establish the provenance of igneous clasts contained in conglomeratic units from different localities (e.g. Spain: Rubio Ordóñez et al. 2004; Morocco: Gigliuto et al. 2004; Argentina: Gallastegui et al. 2014).

In each diagram data from the analysed samples (black dots) are plotted together with those from Sardinia and Calabria basement rocks (coloured shaded areas). The latter geochemical data have been taken from Ferla (1978), Ioppolo \& Puglisi (1980), D'Amico et al. (1981), Messina et al. (1988, 1991), Messina, Russo \& Lima (1993) and Fiannacca et al. (2015) in the case of the calabrian rocks and from Di Simplicio et al. (1974), Del Moro et al. (1975), Traversa \& Vaccaro (1992), Di Vincenzo, Andriessen \& Ghezzo (1996), Cortesogno et al. (1998), Barbey et al. (2008), Cruciani et al. (2013) in the case of Sardinia.

The results have been plotted on TAS (Cox, Bell \& Pankhurst, 1979; Le Bas et al. 1986) and P-Q diagrams (Debon \& LeFort, 1983; diagram based on major elements composition and equivalent to the QAP) in order to make a geochemical classification of the studied samples.

\section{6.a. Classification}

The analysed samples from the Central-Southern Apennines are made of different lithologies, as shown in the petrography section. This variation in the composition is also observed in their geochemical features, summarized in the diagrams of Figures $5 \mathrm{c}, \mathrm{d}, 7-10$. Intrusive and subvolcanic rocks have been plotted separately in order to better evidence their characteristics and test their compatibility with analogous rocks from the Palaeozoic basements.

A first approach to the classification includes the use of two different diagrams depending on the nature of the rocks: a major-element-based multicationic scheme for granitoids in a broad sense (Debon \& LeFort, 1983); and the $\mathrm{Nb} / \mathrm{Y}$ versus $\mathrm{Zr} / \mathrm{TiO}_{2}$ diagram (Winchester \& Floyd, 1977) for the subvolcanic porphyritic clasts. Using these diagrams, the granitoid rocks are classified as adamellites and granites (Fig. 5d) with a peraluminous character as expected in most of granites, and particularly in cordieritebearing granites (Clarke, 1995), while the subvolcanic rocks are classified in a compositional range from andesites to rhyolites (Fig. 8a). It is important to evidence the clear dominance of acidic lithotypes. Both the $\mathrm{SiO}_{2}$ versus $\mathrm{FeO}_{\mathrm{t}} / \mathrm{MgO}$ diagram (Miyashiro, 1974) and the alkalis-iron-magnesium (AFM) diagram (Irvine \& Baragar, 1971) indicate that the porphyritic clasts fit well to a calc-alkaline series (Figs 8b, 8c). The Harker variation diagrams (Harker, 1909) (Figs 9, 10) show some correlation between $\mathrm{SiO}_{2}$ and major elements (Fig. 9a, b). In particular, $\mathrm{SiO}_{2}$ correlates well with $\mathrm{Al}_{2} \mathrm{O}_{3}, \mathrm{MgO}, \mathrm{CaO}, \mathrm{TiO}_{2}$ and $\mathrm{FeO}_{\mathrm{t}}$. On the contrary, plotting $\mathrm{SiO}_{2}$ versus trace elements (Fig. 10a, b) shows much more scattering and no clear correlations can be established. The meaning of these variations is beyond the scope of this work and will not be discussed.

The REE normalized patterns (Boynton, 1984) (Figs 7d, 8d) have been used as a further element to better characterize the collected samples and test their compatibility with the hypothesized source areas. Both intrusive and subvolcanic rocks show similar trends with enrichment in light REEs (LREEs), a marked $\mathrm{Eu}$ negative anomaly and almost flat heavy REEs (HREEs). This is a common pattern for many granitic rocks (e.g. Gallastegui et al. 2014; Dauphas \& Pourmand, 2015). An Ho negative anomaly can be noted for both groups; its significance and the possible explanations are beyond the scope of this work, however.

Each diagram shows the possible geochemical compatibility between the igneous exotic clasts from the 

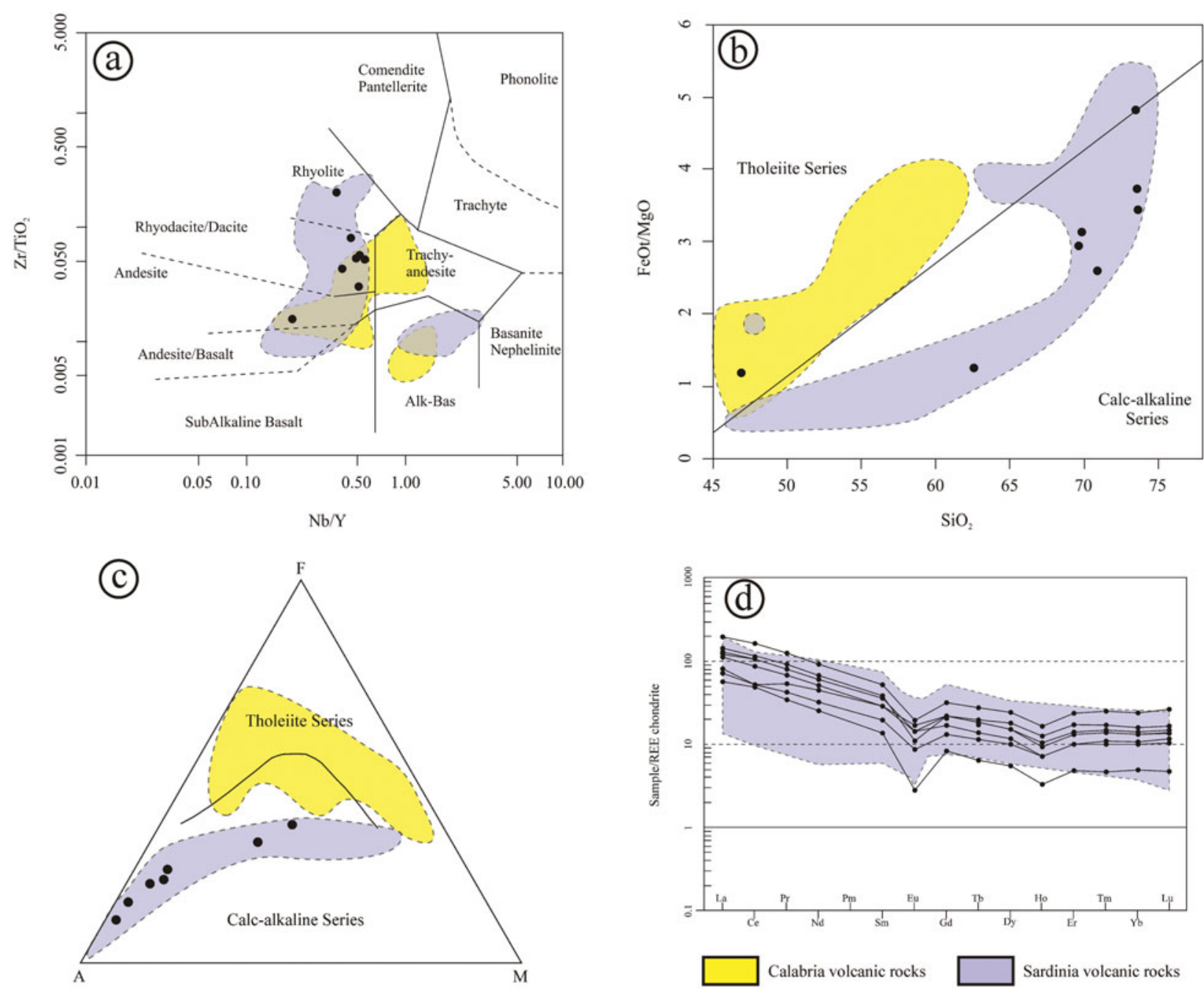

Figure 8. (Colour online) Geochemical features of the analysed porphyritic samples. (a) $\mathrm{Zr} / \mathrm{TiO}_{2} \mathrm{v} . \mathrm{Nb} / \mathrm{Y}$ diagram (Winchester \& Floyd, 1977); (b) FeOt/MgO v. $\mathrm{SiO}_{2}$ diagram (Miyashiro, 1974); (c) AFM diagram (Irvine \& Baragar, 1971). (d) REE normalized patterns (Boynton, 1984). The shaded areas indicate the field of existence of porphyritic rocks from the two possible source areas. Yellow: Calabria; violet: Sardinia (data taken from the literature, see text). No data are available for REE patterns of porphyritic rocks from Calabria.

Central-Southern Apennines and the Calabria and Sardinia basement rocks. The analysed samples (black dots) are plotted together with the compositional fields of both source regions (shaded coloured areas). It is possible to note that no clear discriminations exist for the igneous clasts between Sardinia and Calabria, since the respective fields overlap and no distinct groups can be made (Figs 7, 9a, 10a).

On the contrary, a net discrimination can be made on the subvolcanic analysed samples since Sardinia and Calabria basement rocks plot in clearly separated regions of the diagrams (Figs 8, 9b, 10b). These results and their implications are extensively discussed in the following section.

\section{Discussion: comparison with Sardinian and Calabrian basement rocks}

The petrography and geochemistry of the exotic clasts collected in four conglomeratic units of the CentralSouthern Apennines indicate that their composition is dominantly acidic, with only one sample showing a more basic composition. Despite the relatively homogeneous composition the geochemical features are quite varied, indicating provenances from magmatic bodies with different characteristics.

In order to test the compatibility of the collected samples with the Sardinia and Calabria magmatic basement units and discriminate between them, we analysed the rocks separately according to their main features; in particular, a separation was made between intrusive and subvolcanic porphyritic lithologies.

The diagrams show that no real geochemical distinction exists between the Sardinia and Calabria intrusive rocks. This is not a surprising result, since the two areas shared a common late Variscan evolution and were part of the same block before the Burdigalian drifting (Boccaletti \& Guazzone, 1974; Alvarez, 1976; Minzoni, 1995; Vai, 2001). This common evolution lasted at least until Oligocene time when the opening of the Liguro-Provençal Basin separated these two 

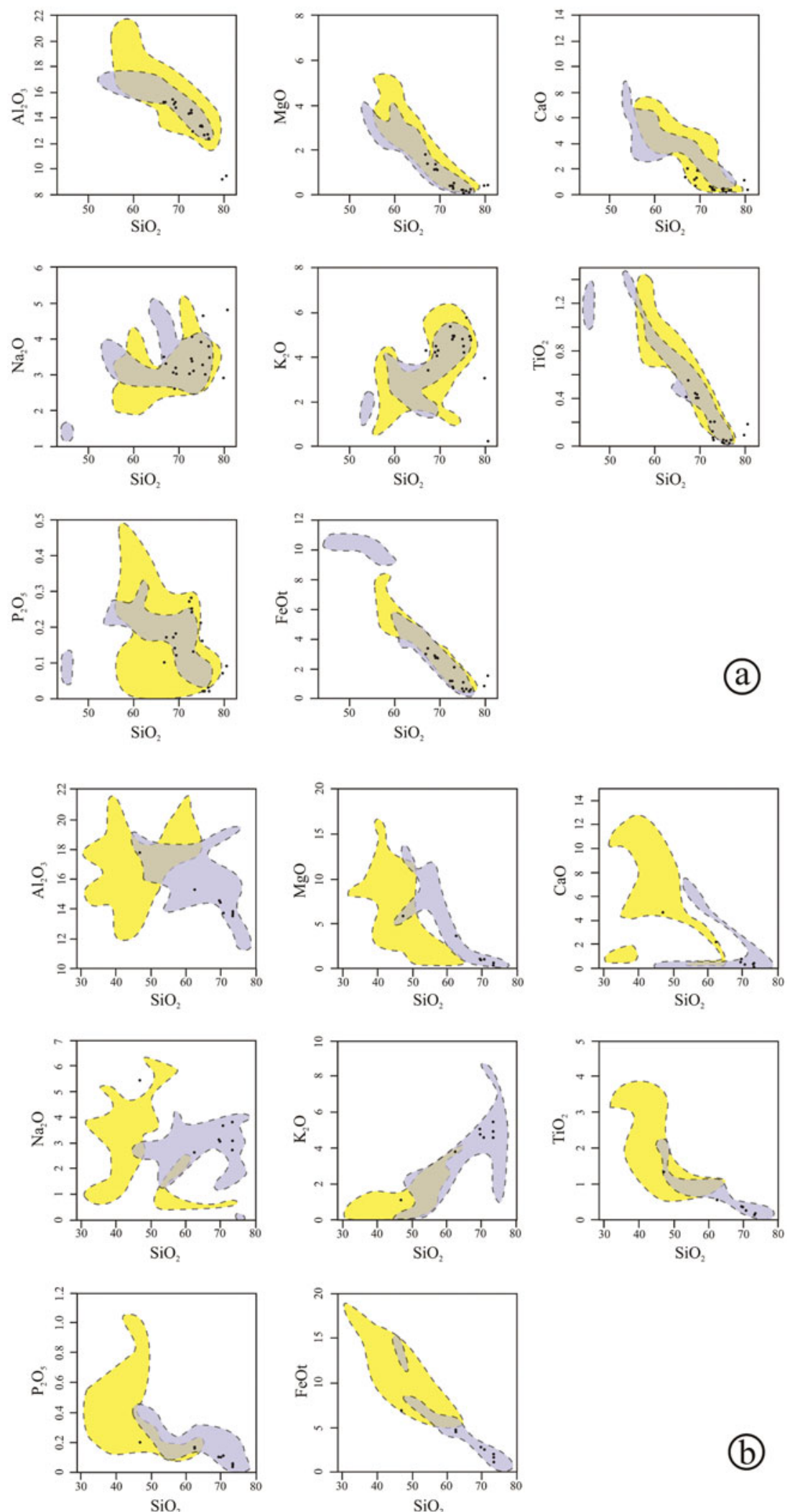

(b)

Figure 9. (Colour online) Harker diagrams of major elements $\left(\mathrm{Al}_{2} \mathrm{O}_{3}, \mathrm{MgO}, \mathrm{CaO}, \mathrm{Na}_{2} \mathrm{O}, \mathrm{K}_{2} \mathrm{O}, \mathrm{TiO}_{2}, \mathrm{P}_{2} \mathrm{O}_{5}, \mathrm{FeOt}\right.$ ) v. $\mathrm{SiO}$ : (a) intrusive samples and (b) porphyritic subvolcanic samples. The shaded areas indicate the field of existence of basement rocks from the two possible source areas. Yellow: Calabria; violet: Sardinia (data taken from the literature, see text).

blocks from the French-Spanish coast (e.g. Alvarez, 1976; Speranza et al. 2002; Advokaat et al. 2014). The later (Langhian: Kastens \& Mascle, 1990; Sartori et al. 2004) opening of the Tyrrhenian Sea determined the separation between Sardinia and Calabria, and the latter reached its present-day position.

Evidence of this common geological evolution also exists in the sedimentary cover of Calabria (e.g. 

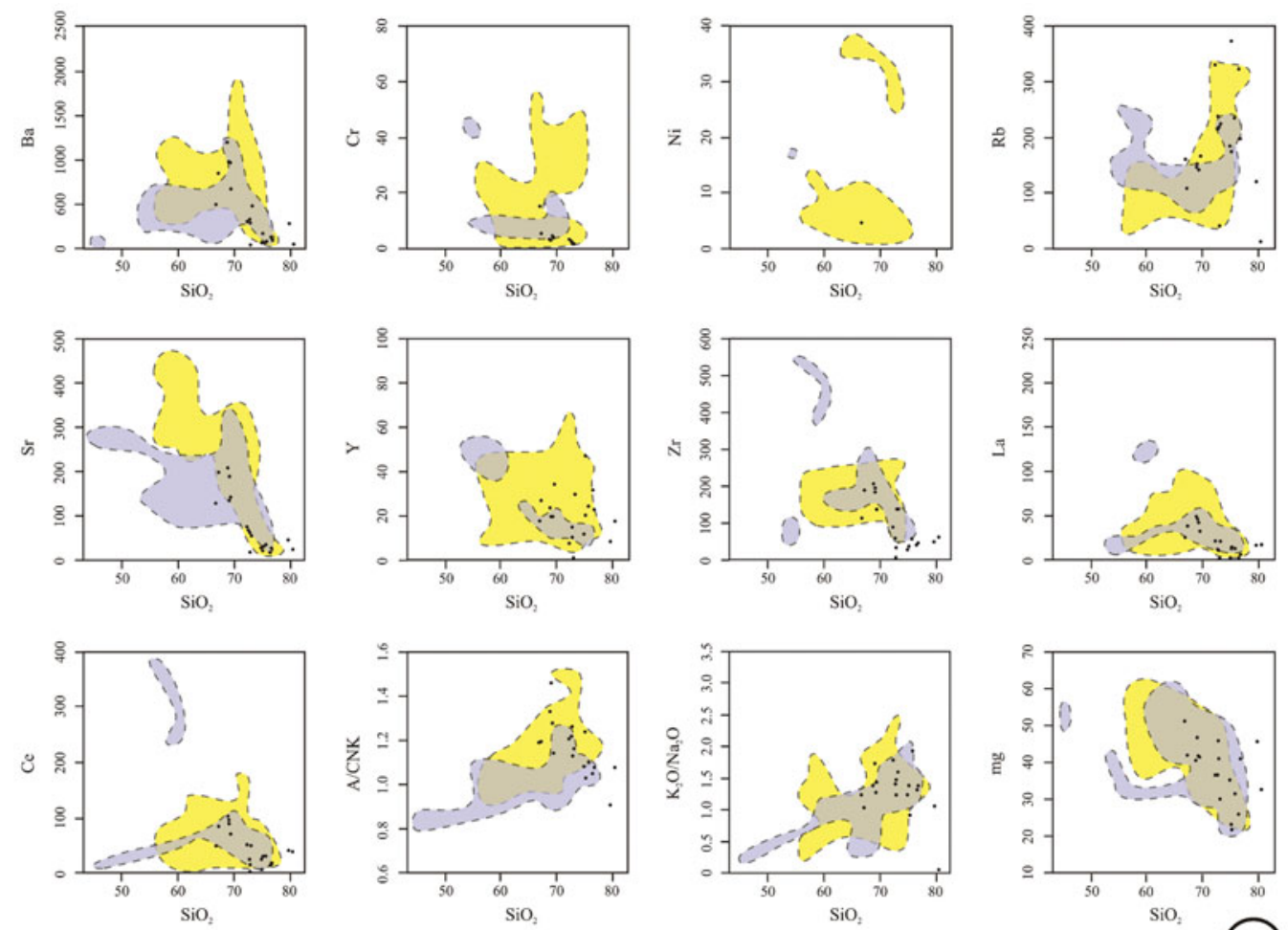

(a)
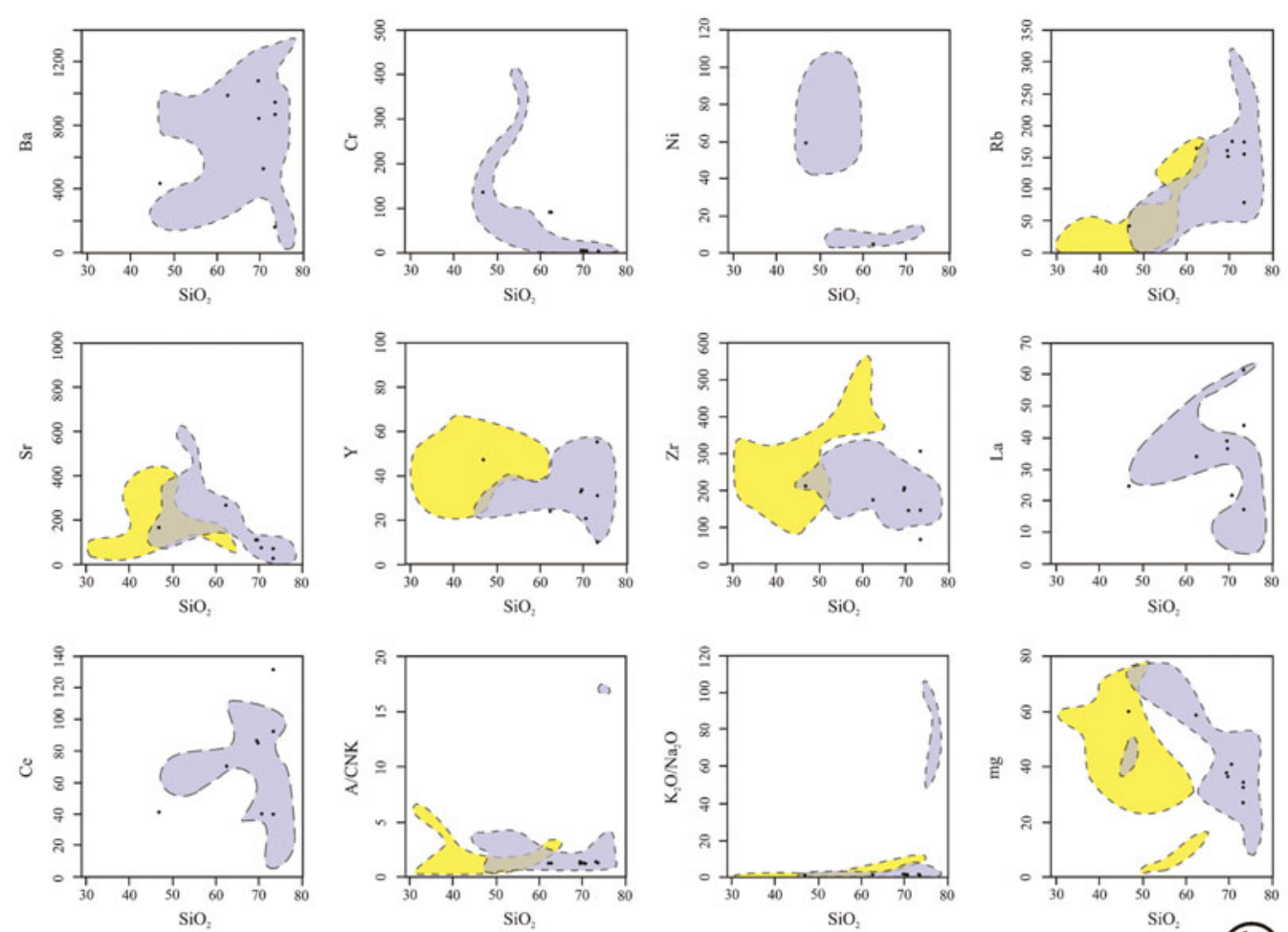

(b)

Figure 10. (Colour online) Harker diagrams of trace elements v. $\mathrm{SiO}_{2}$ : (a) intrusive samples and (b) porphyritic subvolcanic samples. The shaded areas indicate the field of existence of basement rocks from the two possible source areas. Yellow: Calabria; violet: Sardinia (data taken from the literature, see text). No data are available for $\mathrm{Ba}, \mathrm{Cr}, \mathrm{Ni}, \mathrm{La}$ and $\mathrm{Ce}$ composition of porphyritic rocks from Calabria.

Amodio-Morelli et al. 1976; Santantonio \& Teale, 1987; Passeri et al. 2014) that shows analogies with East Sardinia carbonate successions (Dieni et al. 1983; Dieni \& Massari, 1986b; Arragoni et al. 2016).
The comparison between Sardinia (Poli, Ghezzo \& Conticelli, 1989; Di Vincenzo, Andriessen \& Ghezzo, 1996) and Calabria (Atzori, Pezzino \& Rottura, 1977; D'Amico et al. 1981; Del Moro et al. 1982; 
Messina et al. 1991; Fiannacca et al. 2008) cordieritebearing granites yielded the same results, with no clear petrographic and geochemical discrimination between them. This again indicates the common evolution of these two crustal blocks until Oligocene time. Nonetheless the abundance of cordierite-bearing lithotypes in the collected samples (about $30 \%$ of the collected igneous clasts) indicates the relative proximity of at least one cordierite-bearing intrusive body. Similar rocks are also present in Tuscany (e.g. Taylor \& Turi, 1976; Peccerillo \& Donati, 2003) but their relatively young age and geographical position preclude them as a source of the studied conglomeratic units, in particular the Monte Sacro Conglomerates of Cilento.

Considering only the intrusive clasts, their petrographic and geochemical features do not allow any discrimination between Sardinia and Calabria as source areas. The studied samples from the Central-Southern Apennines are compatible with both basements and there is no clear distinction among them.

On the contrary, the comparison between the analysed porphyritic clasts from the Cilento area and the analogous rocks of the Sardinia basement show a good petrographic and geochemical compatibility which is not evident with the Calabria porphyritic rocks. In particular, the analysed conglomerate clasts and the Sardinia porphyritic rocks present a similar geochemical signature (Fig. 8b, 8c). Also the relations between $\mathrm{SiO}_{2}$ and major and trace elements (as shown in the Harker diagrams of Figs 9b, 10b) and the REE patterns (Fig. 8d) show this geochemical affinity (although REE data for Calabria are not available).

We did not analyse the age of the collected samples and the related magmatic bodies. They may have been emplaced in the Sardinia and Calabria basements in distinct phases from the end of the Variscan Orogeny (e.g. Lombardi, Cozzupoli \& Nicoletti, 1974; Ferla, 1978; Cozzupoli et al. 1984; Buzzi, Gaggero \& Oggiano, 2008) to Eocene time (Alpine tectonic phase in Sardinia; Arragoni et al. 2016). Nonetheless, the presented geochemical affinity may suggest Sardinia as the primary source area for the porphyritic clasts of the Monte Sacro Conglomerates of Cilento. Given the similar sedimentary features (dimensions and degree of roundness and alteration), this is also valid for the analysed intrusive clasts.

A further element to identify the source area is provided by the total composition and sedimentary characteristics of the studied conglomerates. The dimensions of the clasts of the Cilento conglomerates and their sedimentary features indicate a short travel distance. As a consequence, the composition of the Cilento conglomerates is directly related to the lithologies cropping out in the source area. The relative volumetric proportions of the clasts of the conglomerates seem to be compatible with the Eastern Sardinia area where an analogous suite of rocks crops out, namely: granitoids (also cordierite-bearing granites, e.g. the San Basilio intrusion: Di Vincenzo, Andriessen \& Ghezzo, 1996); subvolcanic acidic rocks (dacitic and rhyolitic porphyries); metamorphic units (dominantly phyllites and schists compatible with the clasts present in the Monte Sacro Conglomerates, 'Filladi Grigie del Gennargentu' Formation: Oggiano \& Di Pisa, 2001); and sedimentary units, in particular Mesozoic carbonate-platform limestones (Monte $\mathrm{Tu}$ lui and Monte Bardia formations; Amadesi et al. 1960; Dieni \& Massari, 1986b).

Eastern Sardinia may therefore represent the primary source area in Cenozoic time for the conglomerates of the Cilento region. The debated age of these formations (e.g. SGN, 1969; Martelli \& Nardi, 2005) may relate to possible resedimentation or mere geometric relations, and does not affect the results of this study. This is in good agreement with the presence of an uplifting Cenozoic fold-and-thrust belt (Arragoni et al. 2016). The erosion of this growing orogen would provide the mixed sedimentary and metamorphic/igneous material that is currently contained in the Pollica, San Mauro and Monte Sacro Conglomerates formations (Fig. 11a). As evidenced in Section 5 'Sedimentary features and petrography', there is a clear increase of the metamorphic and igneous clasts with respect to the sedimentary ones towards the top of the succession of the Monte Sacro Conglomerates. The litharenite composition of their matrix (Fig. 5a) indicates the less mature character of these deposits compared to the other studied conglomerates (Filettino, Gavignano and Ariano Irpino sedimentary units).

All these features indicate the progressive dismantling of an uplifting orogen, in which a dominantly carbonate sedimentary cover overlies a Palaeozoic basement strongly involved in the compressional deformation. This detritus would feed a sedimentary basin located eastwards of the mountain chain (in present-day coordinates; Fig. 11a), whose succession records the inverse stratigraphy of the orogen. Palaeocurrent directions in the San Mauro and Monte Sacro Conglomerates formations show a dominant provenance from the western and southwestern sectors (in present-day coordinates; Cocco, De Magistris, \& De Pippo, 1978; De Pippo \& Valente, 1991; Valente, 1991; Ciarcia, Vitale \& Lo Schiavo, 2011), which is in good agreement with this interpretation. A sedimentary unit with a similar geodynamic significance is represented by the Cuccuru 'e Flores Conglomerates (Dieni \& Massari, 1965; Dieni, Massari \& Médus, 2008; Oggiano et al. 2011) of the Golfo di Orosei region (Eastern Sardinia). Their composition testifies the erosion and dismantling of the uplifting Cenozoic fold-and-thrust belt of Eastern Sardinia (Arragoni et al. 2016).

The age and sedimentary features of the analysed conglomeratic units indicate that the Cilento conglomerates are the oldest and that they were directly connected to the Eastern Sardinia source area. On the other hand, the features of the Ariano Irpino deposits indicate the recycling of a conglomeratic unit similar to the Cilento unit. This is evidenced by the lesser proportion of igneous and metamorphic clasts, which show a 


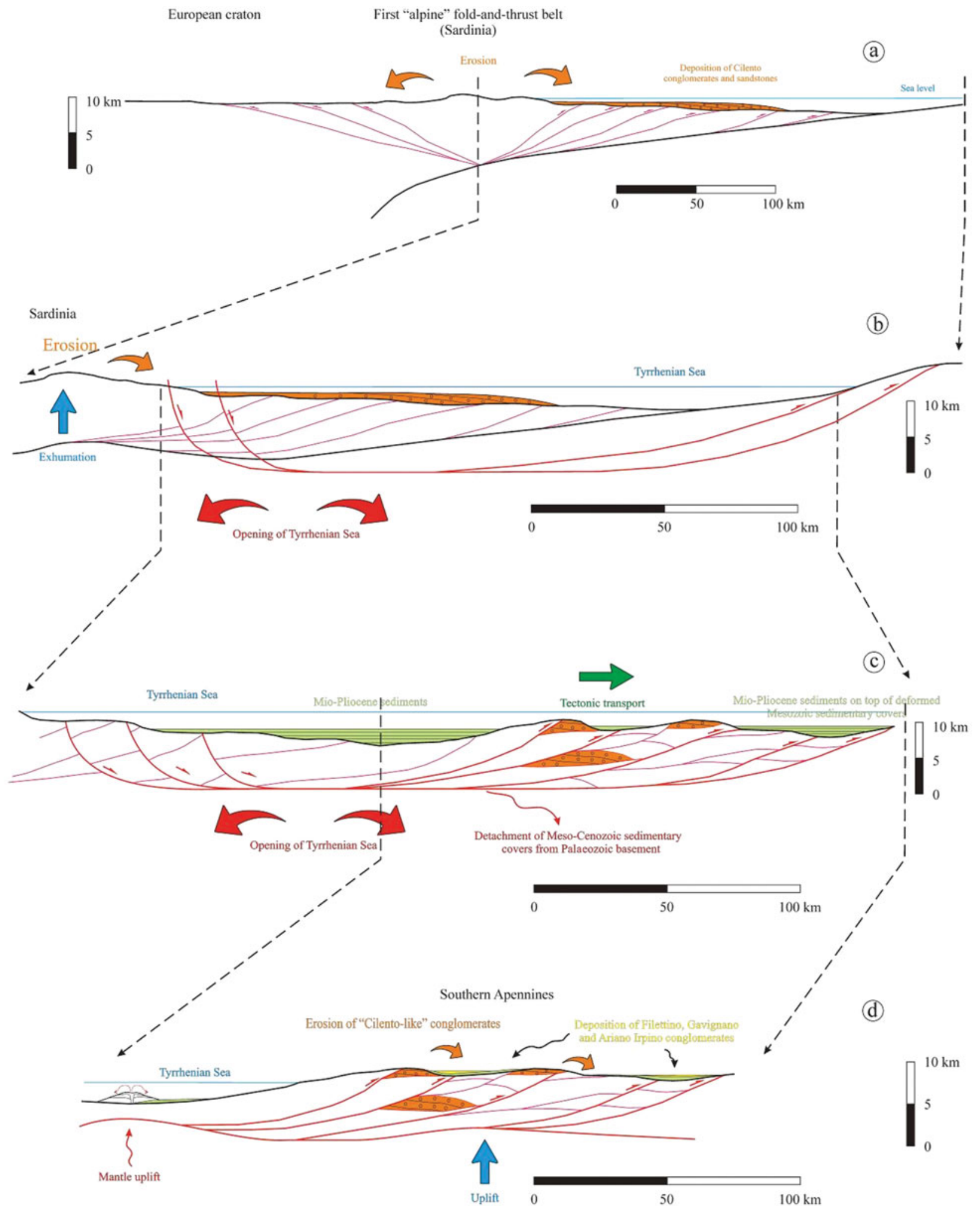

Figure 11. (Colour online) Interpretative sketch of the studied conglomeratic units. (a) Erosion of the uplifting Eastern Sardinia fold-and-thrust belt and deposition of the Cilento conglomerates containing exotic basement clasts (Cenozoic). (b) Opening of the Tyrrhenian Sea and eastwards (in present-day coordinates) translation of the Cilento units on top of deformed Meso-Cenozoic sedimentary units (Langhian). (c) Second phase of deformation involving the Meso-Cenozoic sedimentary units detached from their basement and the Cilento conglomerates. Miocene sediments accumulate unconformably on top of the previous units (upper Miocene - Pliocene). (d) Uplift of the Southern Apennines. The erosion and recycling of Cilento conglomerates and analogous deposits determine the deposition of Filettino, Gavignano and Ariano Irpino conglomerates. 
higher degree of rounding and weathering and a finer average grain size, and by the presence of conglomerate clasts. All these features suggest that the source area contained an older conglomerate unit made of basement clasts, which was subjected to erosion and cannibalization in a continental depositional setting (Fig. 11b, c, d). A significant clast input from the sedimentary units (mainly limestones and sandstones) of the Ariano Irpino substrate resulted in a lowered proportion of exotic clasts.

The Filettino and Gavignano units, in turn, would represent a different stage of reworking. In these units, the exotic basement clasts are almost absent due to their destruction by post-depositional weathering before they could be transported into the basin and the composition of the succession is dominated by sedimentary clasts, mainly derived from the surrounding Mesozoic carbonate units. Exotic material is represented by the abundant sandstone clasts and the rare pelagic-limestone clasts contained in the conglomerates. These are the product of the dismantling of pelagic sedimentary units, which cannot be clearly identified today but which should lie in a more westwards position (in present-day coordinates) with respect to the current outcrops, as suggested by the palaeocurrent indications.

The matrix of the Filettino and Gavignano conglomeratic units indicates a provenance from an area containing metamorphic and igneous rocks, as evidenced by the presence of abundant, almost euhedral feldspars and crystalline lithic fragments. These particles are interpreted to result from the breaking down of the metamorphic and igneous clasts of the gravels present in the previous basin, now under recycling. The recycling of this previous basin constitutes a mixed source, yielding the basement clasts of its gravels and Mesozoic sedimentary, mainly calcareous, clasts from its substrate. The bimodality of the quartz grains suggests a possible double provenance from crystalline rocks and pre-existing sandstones. Quartz grains deriving from sandstone recycling would be finer grained on average due to their polycyclic origin.

This allows us to conclude that the Cilento conglomerates would represent a primary deposit, directly fed by the Eastern Sardinia basement rocks. On the contrary, the Ariano Irpino, Filettino and Gavignano units represent secondary deposits, formed by the erosion and mixing between in situ sedimentary units and exotic clasts from the dismantling of pre-existing conglomerates and gravels which contained metamorphic and igneous clasts.

The geodynamic implications of these results are noteworthy since they suggest a link between the Cilento area, and subsequently the Central-Southern Apennines Meso-Cenozoic rocks, and the Cenozoic Eastern Sardinia fold-and-thrust belt.

In our study, sedimentary, petrographic and geochemical results suggest the proximity of the European continent to the Central-Southern Apennines carbonate-platform successions at least after the form- ation of the Alpine collisional belt, whose erosion produced the studied exotic clasts.

The successive opening of the Tyrrhenian Sea determined the fragmentation of the pre-existing Alpine chain and the separation between Sardinia, Calabria and the Central-Southern Apennines (Fig. 11b). During this second phase of deformation, the MesoCenozoic sedimentary cover of the Central-Southern Apennines was detached from its basement and involved in a further compressional event which determined its current structural location and final assembly (Fig. 11c, d). In this way, the studied conglomeratic deposits, directly connected and fed by the Sardinian orogen, participated in this detachment and became incorporated in the Apenninic chain as evidence of the dismantled Alpine chain.

\section{Conclusions}

The study of the sedimentary, petrographic and geochemical features of the exotic clasts from the conglomeratic units of the Central-Southern Apennines provides new evidence for the reconstruction of the geodynamic evolution of the Central Mediterranean area in Cenozoic time. The presence of exotic basement clasts in areas where only Meso-Cenozoic sedimentary rocks crop out is an important element to link the geological evolution of the Central-Southern Apennines to Sardinia.

Petrographic, geochemical and sedimentary analyses indicate a compatibility among these exotic clasts and a source area located approximately in present-day Eastern Sardinia. This is also confirmed by the average composition of the conglomerates, which show a suite of rocks typical of an uplifting orogen compatible with that recognized by Arragoni et al. (2016) in the Golfo di Orosei region (Eastern Sardinia).

The sedimentary features of the four studied conglomeratic units allow a primary (i.e. directly fed by the Sardinian source area) conglomerate, represented by the Cilento Unit, and secondary conglomerates, derived from the erosion and reworking of older conglomeratic units bearing exotic basement clasts (Fig. 11), to be recognized.

The proximity of the studied conglomeratic units to the European continent evidenced by the presented results, and the lack of Meso-Cenozoic sedimentary units at the bottom of the Tyrrhenian Sea (where Mio-Quaternary sediments lie directly on the Variscan basement: DSDP, 1978; Sartori et al. 2004) may imply that a part of the carbonate platforms of the CentralSouthern Apennines was located on the European margin before the opening of the Tyrrhenian Sea. These Meso-Cenozoic units underwent a first phase of deformation during Eocene-Oligocene time, resulting in a double-verging orogen whose relics are preserved in Eastern Sardinia (Golfo di Orosei region; Fig. 11a). The consequent uplift of this Cenozoic Alpine orogen led to strong erosion and the accumulation of sedimentary units such as the Cilento conglomerates. 
The opening of the Tyrrhenian Sea since Langhian time split apart this first fold-and-thrust chain, leading to the detachment of its cover formed by the thrusted units of the European and African passive margins to form the present-day Apenninic chain.

The Cilento conglomerates were fed by the erosion of the former fold-and-thrust Alpine chain and were detached and thrusted on the eastern side of the Tyrrhenian Basin together with the underlying carbonateplatform units (Fig. 11b, c). Erosion and reworking of these conglomerates and other analogous units formed re-deposited conglomerates such as the Filettino, Gavignano and Ariano Irpino units (Fig. 11d).

These results add new robust evidence for understanding the complex geodynamic evolution of the Central Mediterranean since early Cenozoic time, and provide new clues for the reconstruction of the relations between the European and African margins with the development of the Alpine fold-and-thrust belt. The studied exotic clasts represent the relics of this chain.

\section{References}

Accordi, G., Carbone, F., La Monica, G. B., Praturlon, A., Sirna, G. \& Zalaffi, M. 1969. Il Neogene nell'Appenino laziale-abruzzese. Giornale di Geologia 35, 235-65.

Acton, C. E., Priestley, K., Mitra, S. \& Gaur, V. K. 2010. Crustal structure of the Darjeeling - Sikkim Himalaya and southern Tibet. Geophysical Journal International 184(2), 829-52, doi:10.1111/j.1365246X.2010.04868.x.

Advokat, E. L., van Hinsbergen, D. J. J., Maffione, M., Langereis, C. G., Vissers, R. L. M., Cherchi, A., Schroeder, R., Madani, H. \& Columbu, S. 2014. Eocene rotation of Sardinia, and the paleogeography of the western Mediterranean region. Earth and Planetary Science Letters 401, 183-95, doi:10.1016/j.epsl.2014.06.012.

Alberti, U., Bergomi, C., Catenacci, V., Centamore, E., Cestari, G., Chiocchini, M., Chiocchini, U., Manganelli, V., Molinari-Paganelli, V., PanseriCrescenzi, C., Salvati, L. \& Tilia-Zuccari, A. 1975. Note illustrative della carta geologica d'Italia alla scala 1:50.000. Foglio 389 - Anagni Servizio Geologico d'Italia, Litografia Artistica Cartografica, LAC, Firenze.

Alvarez, W. 1976. A former continuation of the Alps. Geological Society of America Bulletin 87, 891-96, doi:10.1130/0016-7606(1976)87.

Alvarez, W. 1991. Tectonic evolution of the CorsicaApennines-Alps region studied by the method of successive approximations. Tectonics 10(5), 936-47, doi:10.1029/91TC00232.

Amadesi, E., Cantelli, C., Carloni, G. C. \& Rabbi, E. 1960. Ricerche geologiche sui terreni sedimentari del foglio 208 Dorgali. Giornale di Geologia 28, 59-87.

Amodio-Morelli, L., Bonardi, G., COlOnNA, V., Dietrich, D., Giunta, G., Ippolito, F., Liguori, V., Lorenzoni, S., Paglionico, A., Perrone, V., Piccarreta, G., Russo, M., Scandone, P., ZantettinLoRENZONi, E. \& ZupPeTtA, A. 1976. L'arco CalabroPeloritano nell'orogene appenninico-maghrebide. Memorie Della Società Geologica Italiana 17, 1-60.
Amore, F. O., Basso, C., Ciarcia, S., Di Nocera, S. Matano, F., Torre, M., Ciampo, G., Di Donato, V., Esposito, P. \& Staiti, D. 1998. Nuovi dati stratigrafici sul Pliocene affiorante tra il fiume Ufita e il torrente Cervaro (Irpinia, Appennino Meridionale). Bollettino della Società Geologica Italiana 117, 455-66.

Amore, F. O., Bonardi, G., Ciampo, G., De Capoa, P., Perrone, V. \& Sgorsso, I. 1988a. Relazioni tra "Flysch interni" e domini appenninici: reinterpretazione delle formazioni di Pollica, San Mauro e Albidona nel quadro della evoluzione inframiocenica delle zone esterne appenniniche. Memorie Della Società Geologica Italiana 41, 285-97.

Amore, F. O., Ciampo, G., De Capoa, P. \& RuggieroTAdDEI, E. 1988b. Problemi biostratigrafici dei sedimenti terrigeni nell'Appennino Centro-Meridionale. Memorie Della Società Geologica Italiana 41, 621-25.

Angelucci, A. \& Devoto, G. 1966. Geologia del Monte Caccume (Frosinone). Geologica Romana V, 177-96.

Angelucci, A., Devoto, G. \& Farinacci, A. 1963. Le "argille caotiche" di Colle Cavallaro a est di Castro dei Volsci (Frosinone). Geologica Romana II, 30529.

Arragoni, S., Maggi, M., Cianfarra, P. \& Salvini, F. 2016. The Cenozoic fold-and-thrust belt of Eastern Sardinia: evidences from the integration of field data with numerically balanced geological cross-section. Tectonics 35(6), 1404-22, doi:10.1002/2015TC004004.

Arragoni, S., Martini, I. \& SANDrelli, F. 2012. Facies associations map of the Pliocene marine deposits of the central-southern Siena Basin (Tuscany, Italy). Journal of Maps 8(4), 406-12, doi:10.1080/17445647.2012.744706.

Atzori, P., Pezzino, A. \& Rottura, A. 1977. La massa granitica di Cittanova (Calabria Meridionale): relazioni con le rocce granitoidi del Massiccio delle Serre e con le metamorfiti di Canolo, S. Nicodemo e Molochio (nota preliminare). Bollettino della Società Geologica Italiana 96, 387-91.

Bally, A. W., Gordy, P. L. \& Stewart, G. A. 1966. Structure, seismic data and orogenic evolution of Southern Canadian Rocky Mountains. Bulletin of Canadian Petroleum Geology 14(3), 337-81.

Barattolo, F. \& Parente, M. 1991. Late Cretaceous - Paleogene of Roccadaspide (Salerno, Campania). In: Field Trip Guide Book, Proccedings of 5th International Symposium on Fossil Algae (eds F. Barattolo, P. De Castro \& M. Parente), Capri, 7-12 April 1991.

Barbey, P., Gasquet, D., Pin, C. \& Bourgeix, A. L. 2008. Igneous banding, schlieren and mafic enclaves in calcalkaline garnites: the Budduso pluton (Sardinia). Lithos 104, 147-63, doi:10.1016/j.lithos.2007.12.004.

Barchi, M., Landuzzi, A., Minelli, G. \& Pialli, G. 2001. Outer Northern Apennines. In: Anatomy of an Orogen, the Apennines and Adjacent Mediterranean Basins (eds G. B. Vai \& I. P. Martini), pp. 215-54. Dordrecht: Kluver Academic Publishers, Dordrecht, 215-54.

Beneo, E. 1943. Note illustrative alla Carta Geologica d'Italia alla scala 1:100.000, foglio Alatri. Roma: Istituto Poligrafico e Zecca dello Stato, $55 \mathrm{pp}$.

Bigi, G., Cosentino, D., Parotto, M., Sartori, R., Scandone, P., Carrozzo, M. T., Luzio, D., Margiotta, C. \& Quarta, T. 1992. Structural model of Italy and gravity map. CNR-Progetto finalizzato Geodinamica, Quaderni della Ricerca Scientifica, 114.

Biju-Duval, B., Letouzey, J., Montadert, L., Courrier, P., Mugniot, J. F. \& Sancho, J. 1974. Geology of the 
Mediterranean Sea Basins. In: The Geology of Continental Margins (eds C. A. Burk \& C. L. Drake), pp. 695721. Berlin, Heidelberg: Springer.

Boccaletti, M., Ciaranfi, N., Cosentino, D., Deiana, G., Gelati, R., Lentini, F., Masari, F., Moratti, G., Pescatore, T., Ricci Lucchi, F. \& Tortorici, L. 1990. Palinspastic restoration and paleogeographic reconstruction of the peri-Tyrrhenian area during the Neogene. Palaeogeography, Palaeoclimatology, Palaeoecology 77, 41-50, doi:10.1016/0031-0182(90)90097-Q.

Boccaletti, M. \& Guazzone, G. 1974. Remnant arcs and marginal basins in the Cainozoic development of the Mediterranean. Nature 252, 18-21, doi:10.1038/252018a0.

Bonardi, G., Amore, F. O., Ciampo, G., De Capoa, P., Miconnet, P. \& Perrone, V. 1988a. Il Complesso Liguride Auct: stato delle conoscenze e problemi aperti sull'evoluzione pre-appenninica ed i suoi rapporti con l'arco calabro. Memorie Della Società Geologica Italiana 41, 17-35.

Bonardi, G., Cavazza, W., Perrone, S. \& Rossi, S. 2001. Calabria-Peloritani terrane and northern Ionian Sea. In: Anatomy of an Orogen, the Apennines and Adjacent Mediterranean Basins (eds G. B. Vai \& I. P. Martini), pp. 287-306. Dordrecht: Kluver Academic Publishers.

BoynTON, W. V. 1984. Cosmochemistry of the rare earth elements: meteorite studies. In: Rare Earth Element Geochemistry (ed. P. Henderson), pp. 63-114. Amsterdam: Elsevier.

Brogi, A., Lazzarotto, A., Liotta, D. \& CROP 18 Working Group 2005. Structural features of Southern Tuscany and geological interpretation of the CROP 18 Seismic Reflection Survey (Italy). Bollettino della Società Geologica Italiana, Volume Speciale 3, 21336.

Butler, R. W. H. 1986. Thrust tectonics, deep structure and crustal subduction in the Alps and Himalayas. Journal of the Geological Society 143, 857-73, doi:10.1144/gsjgs.143.6.0857.

Buzzi, L., Gaggero, L. \& Oggiano, G. 2008. Lower Permian age of the Santa Giusta Ignimbrite (NW Sardinia): insights on the post-collisional evolution of a sector of European crust between the Lower Permian and Early Triassic. Italian Journal of Geosciences 127, 683-95.

Carmignani, L., Decandia, F. A., Disperati, L., Fantozzi, P. L., Kligfield, R., Lazzarotto, A., Liotta, D. \& MeCCheri, M. 2001a. Inner Northern Apennines. In: Anatomy of an Orogen, the Apennines and Adjacent Mediterranean Basins (eds G. B. Vai \& I. P. Martini), pp. 197-214. Dordrecht: Kluwer Academic Publishers.

Carmignani, L., Decandia, F. A., Fantozzi, P. L., Lazzarotto, A., Liotta, D. \& Meccheri, M. 1994. Tertiary extensional tectonics in Tuscany (Northern Apennines, Italy). Tectonophysics 238, 295-315, doi:10.1016/0040-1951(94)90061-2.

Castellarin, A. \& Transalp Working Group 2004. Structural synthesis of the Eastern Alps: A collisional orogenic chain. In The Geology of Italy (eds U. Crescenti, S. D’Offizi, S. Merlino \& L. Sacchi), pp. 3-13. Special Volume of the Italian Geological Society for the IGC 32, Florence.

Catalano, S., Monaco, C., Tortorici, L., Paltrinieri, W. $\&$ STEEL, N. 2004. Neogene-Quaternary tectonic evolution of the Southern Apennines. Tectonics 23(2), 1-19.

Cathelineau, M. 1986. The hydrothermal alkali metasomatism effects on granitic rocks: quartz dissolution and related subsolidus changes. Journal of Petrology 27, 945-65, doi:10.1093/petrology/27.4.945.
Cavazza, W., Roure, F. \& Ziegler, P. A. 2004. The Mediterranean area and the surrounding regions: active processes, remnants of former Tethyan oceans and related thrust belts. In: The TRANSMED Atlas: The Mediterranean Region from Crust to Mantle (eds W. Cavazza, F. Roure, W. Spakman, G. M. Stampfli \& P. A. Ziegler), pp. 1-29. Berlin, Heidelberg: Springer.

Chiocchini, U. 2007. Note illustrative della carta geologica d'Italia alla scala 1:50.000. Foglio 432 - Benevento. Roma: ISPRA, Roma, 126 pp.

Chiocchini, U. \& Chiocchini, F. 1999. I conglomerati messiniani dell'area tra Benevento e il F. Sabato (Appennino meridionale). Studi Geologici Camerti 14, 255-78.

Cianfarra, P. \& Maggi, M. 2017. Cenozoic extension along the reactivated Aurora Fault System in the East Antarctic Craton. Tectonphysics 703-4, 135-43, doi:10.1016/j.tecto.2017.02.019.

Cianfarra, P. \& Salvini, F. 2014. Ice sheet surface lineaments as nonconventional indicators of East Antarctica bedrock tectonics. Geosphere 10(6), 1411-18, doi:10.1130/GES01074.1.

Ciarcia, S. \& Torre, M. 1996. I ciottoli dei conglomerati medio-pliocenici dell'Appennino campano: provenienza, elaborazione, ambiente di deposizione. Bollettino della Società Geologica Italiana 115, 569-81.

Ciarcia, S., Vitale, S. \& Lo Schiavo, L. 2011. Paleocurrent analysis in the Cilento Group deposits (Campania, southern Italy). Rendiconti Online Societa Geologica Italiana 15, 32-3.

Cieszkowski, M., Malata, E., Oszczypko, N., Pescatore, T., Senatore, M. R., ŚlączKa, A. \& Valente, A. 1992. Rapporti tra le successioni di Monte Sacro e Monte della Stella nel Flysch del Cilento (Appennino meridionale). Rendiconti dell'Accademia di Scienze Fisiche Matematiche in Napoli IV, 69, 3356.

Cieszkowski, M., Oszczypko, N., Pescatore, T., Ślączka, A., Senatore, M. R. \& Valente, A. 1994. Excursion A9: Deep-sea clastic sediments and associated megaturbidites and olistostromes (Cenozoic, Cilento, Southern Italy). In: Pre-meeting Fieldtrip Guidebook, 15th IAS Regional Meeting (eds G. Carannante \& R. Tonielli), pp. 191-220. International Association of Sedimentologists, April 1994, Ischia, Italy.

Cipollari, P. \& Cosentino, D. 1995. Miocene unconformities in the central Apennines: geodynamic significance and sedimentary basin evolution. Tectonophysics $\mathbf{2 5 2}$, 375-89, doi: 10.106/0040-1951(95)00088-7.

Civitelli, G. \& CoRdA, L. 1982. Nuovi dati sulla Pietraforte: sue caratteristiche e rapporti con i flysch calcarei dell'area tolfetana. Geologica Romana 21, 191-216.

Clarke, D. B. 1995. Cordierite in felsic igneous rocks: a synthesis. Mineralogical Magazine 59, 311-25.

Cocco, E. 1971. Note illustrative alla carta geologica d'Italia scala 1:100.000. Foglio 209 - Vallo della Lucania. Servizio Geologico d'Italia, Roma, $47 \mathrm{pp}$.

Cocco, E., De Magistris, M. A. \& De Pippo, T. 1978. Sedimentologia del Flysch del Cilento. 1: le arenarie di Serramezzana (Cilento, Appennino Meridionale). Geologica Romana 17, 289-302.

Cortesogno, L., Cassinis, G., Dallagiovanna, G., Gaggero, L., Oggiano, G., Ronchi, A., Seno, S. \& VAnossi, M. 1998. The Variscan post-collisional volcanism in Late Carboniferous-Permian sequences of Ligurian Alps, Southern Alps and Sardinia (Italy): a synthesis. Lithos 45, 305-28, doi:10.1016/S00244937(98)00037-1. 
Cosentino, D. \& Cipollari, P. 2012. The Messinian Central Apennines. Rendiconti Online Societa Geologica Italiana 23, 45-51.

Cosentino, D., Cipollari, P. \& Pipponzi, G. 2003. Il sistema orogenico dell'Appennino centrale: vincoli stratigrafici e cronologia della migrazione. Studi Geologici Camerti, 2003, 87-101.

Cox, K. G., Bell, J. D., \& Pankhurst, R. J. 1979. The Interpretation of Igneous Rocks. Dordrecht: George Allen \& Unwin, $450 \mathrm{pp}$.

Cozzupoli, D., Gerbasi, G., Nicoletti, M. \& Petrucciani, C. 1984. Età K-Ar delle ignimbriti permiane di Galtellì (Orosei - Sardegna orientale). Rendiconti della Società Italiana di Mineralogia e Petrologia 39, 471-76.

Critelli, S. 1991. Evoluzione delle mode detritiche delle successioni arenitiche terziarie dell'Appenino meridionale. Memorie Della Società Geologica Italiana 38, 601-19.

Critelli, S. \& Le Pera, E. 1990. Composizione e provenienza delle areniti delle formazioni del Torrente Bruca e di Monte Sacro (Cilento, Appennino meridionale). Giornale di Geologia 52(1-2), 121-33.

Critelli, S. \& Le PerA, E. 1994. Detrital modes and provenance of Miocene sandstones and modern sands of the Southern Apennines thrust-top basins (Italy). Journal of Sedimentary Research 64(4), 82435.

Cruciani, G., Franceschelli, M., Musumeci, G., Spano, M. E. \& Tiepolo, M. 2013. U-Pb zircon dating and nature of metavolcanics and metarkoses from the Monte Grighini Unit: new insights on Late Ordovician magmatism in the Variscan belt in Sardinia, Italy. International Journal of Earth Sciences 102, 2077-96, doi:10.1007/s00531-013-0919-z.

D'Agostino, N., Avallone, A., Cheloni, D., D’Anastasio, E., Mantenuto, S. \& Selvaggi, G. 2008. Active tectonics of the Adriatic region from GPS and earthquake slip vectors. Journal of Geophysical Research 113, B12413, doi:10.1029/2008JB005860.

D’Amico, C., Rottura, A., Maccarrone, E. \& Puglisi, G. 1981. Peraluminous granitic suite of Calabria-Peloritani Arc (Southern Italy). Rendiconti Società Italiana di Mineralogia e Petrologia 38(1), 35-52.

D’Argenio, B., Pescatore, T. \& Scandone, P. 1973. Schema geologico dell'Appennino Meridionale (Campania e Lucania). Atti Accademia Lincei, Convegno "Moderne vedute sulla geologia dell'Appennino" 183, 49-72.

D’Argenio, B., Pescatore, T. \& Scandone, P. 1975. Structural pattern of the Campania-Lucania Apennines. Structural Model of Italy, Quaderni Ricerca Scientifica 90, 313, 327.

Dauphas, N. \& Pourmand, A. 2015. Thulium anomalies and rare earth element patterns in meteorites and Earth: Nebular fractionation and the nugget effect. Geochimica et Cosmochimica Acta 163, 234-61, doi:10.1016/j.gca.2015.03.037.

De Pippo, T. \& Valente, A. 1991. Osservazioni sedimentologiche preliminari sui "Conglomerati di Monte Sacro" (Campania). Bollettino della Società Geologica Italiana 110, 47-51.

De Rita, D., Funiciello, R., Pantosti, D., Salvini, F., Sposato, A. \& Velonà, M. 1986. Geological and structural characteristics of the Pontine Islands (Italy) and implications with the evolution of the Tyrrhenian margin. Memorie della Società Geologica Italiana 36, 5565 .
Debon, F. \& LeFort, P. 1983. A chemical-mineralogical classification of common plutonic rocks and associations. Transactions of Royal Society of Edinburgh, Earth Sciences 73, 135-149.

Del Moro, A., Di Simplicio, P., Ghezzo, C., Guasparri, G., Rita, F. \& SABATINI, G. 1975. Radiometric data and intrusive sequence in the Sardinian Batholith. Neues Jahrbuch für Mineralogie Abhandlungen 126, 28-44.

Del Moro, A., Pardini, G., Maccarrone, E. \& Rottura, A. 1982. Studio radiometrico $\mathrm{Rb}-\mathrm{Sr}$ di granitoidi peraluminosi dell'arco calabro-peloritano. Rendiconti Società Italiana di Mineralogia e Petrologia 38(3), 101526.

Devoto, G. 1967a. Sguardo geologico dei Monti Simbruini (Lazio nord-orientale). Geologica Romana IX, 12736.

Devoto, G. 1967b. Note geologiche sul settore centrale dei Monti Simbruini ed Ernici (Lazio nord-orientale). Bollettino della Società dei Naturalisti in Napoli 76, 76112.

Di Girolamo, P., Morra, V. \& Perrone, V. 1991. Ophiolitic olistoliths in middle Miocene turbidites (Cilento Group) at Mount Centaurino (southern Apennines, Italy). Ofioliti 17, 199-217.

Di Girolamo, P., Sgrosso, I., De Gennaro, R. \& Giurazzi, S. 2000. Metamorphic rocks in Campania (Southern Italy): the "Mondragone Marbles". Bollettino della Società Geologica Italiana 119(3), 761-6.

Di Simplicio, P., Ferrara, G., Ghezzo, C., Guasparri, G., Pellizzer, R., Ricci, C. A., Rita, F. \& Sabatini, G. 1974. Il metamorfismo e il magmatismo Paleozoico nella Sardegna. Rendiconti Società Italiana di Mineralogia e Petrologia XXX, 979-1068.

Di Vincenzo, G., Andriessen, P. A. M. \& Ghezzo, C. 1996. Evidence of two different components in a Hercynian peraluminous cordierite-bearing granite: the San Basilio intrusion (Central Sardinia, Italy). Journal of Petrology 37(5), 1175-206.

Dickinson, W. R., Beard， L. S., BraKenridge，G. R., Erjavec, J. L., Ferguson, R. C., Inman, K. F., KNePP, R. A., LindBerG, F. A. \& RYBERG, P. T. 1983. Provenance of North American Phanerozoic sandstones in relation to tectonic setting. GSA Bulletin 94, 222-35, doi:10.1130/00167606(1983)94<222:PONAPS $>2.0 . C O ; 2$.

Dieni, I., Fischer, J. C., Massari, F., SAlardCheboldaefF, M. \& Vozenin-Serra, C. 1983. La succession de Genna Selole (Baunei) dans le cadre de la palégéographie mésojurassique de la Sardaigne orientale. Memorie di Scienze Geologiche, Università di Padova 36, 117-48.

Dieni, I. \& MASSARI, F. 1965. Precisazioni sull'età di alcuni conglomerati affioranti presso Siniscola, Orosei e Dorgali (Sardegna orientale). Rendiconti Accademia Nazionale dei Lincei 40, 205-11.

DienI, I. \& MASSARI, F. 1986b. Mesozoic of Eastern Sardinia. In: 19th Micropaleontological Colloquium Guidebook (ed. A. Cherchi), pp. 66-78. Sardinia, Italy.

Dieni, I., Massari, F. \& Médus, J. 2008. Age, depositional environment and stratigraphic value of the Cuccurue Flores Conglomerate: Insight into the Palaeogene to Early Miocene geodynamic evolution of Sardinia. Bulletin de la Societe Geologique de France 179(1), 51-72, doi:10.2113/GssGfbull.179.1.51.

Dietrich, D. \& SCANDONE, P. 1972. The position of basic and ultrabasic rocks in the tectonic units of the Southern Apennine. Atti dell'Accademia Pontaniana in $\mathrm{Na}$ poli 21, 61-75. 
DiLEK, Y. 2006. Collision tectonics of the Mediterranean region: Causes and consequences. In: Postcollisional Tectonics and Magmatism in the Mediterranean Region and Asia (eds Y. Dilek \& S. Pavlides), pp. 113. Geological Society of America, Special Paper 409, doi:10.1130/2006.2409(01).

DSDP 1978. Initial report of the Deep Sea Drilling Project, 42, doi:10.2973/dsdp.proc.42-1.1978. Texas A \& M University, Ocean Drilling Program, College Station, Texas, USA.

Favali, P., Funiciello, R., Mattietti, G., Mele, G. \& SAlvini, F. 1993. An active margin across the Adriatic Sea (central Mediterranean Sea). Tectonophysics 219, 109-17, doi:10.1016/0040-1951(93)90290-Z.

FerLA, P. 1978. Natura e significato geodinamico del vulcanismo preercinico presente nelle filladi e nei semiscisti dei Monti Peloritani (Sicilia). Rendiconti Società Italiana di Mineralogia e Petrologia 34(1), 55-74.

Fiannacca, P., Cirrincione, R., Bonanno, F. \& Carciotto, M. M. 2015. Source-inherited compositional diversity in granite batholiths: the geochemical message of Late Paleozoic intrusive magmatism in central Calabria (southern Italy). Lithos 236, 123-40, doi:10.1016/j.lithos.2015.09.003.

Fiannacca， P., Williams, I. S., Cirrincione, R. \& Pezzino, A. 2008. Crustal contributions to Late Hercynian peraluminous magmatism in the southern Calabria-Peloritani Orogen, Southern Italy: petrogenetic inferences and the Gondwana connection. Journal of Petrology 49(8), 1497-514, doi:10.1093/petrology/egn035.

Fitz-Diaz, E., Hudleston, P. \& Tolson, G. 2011. Comparison of tectonic styles in the Mexican and Canadian Rocky Mountains fold-thrust belt. In: Kinematic Evolution and Structural Styles of Fold-and-Thrust Belts (eds J. Poblet \& R. J. Lisle), pp. 149-67. Geological Society, London, Special Pubblication no. 349.

Gallastegui, G., GonZÁlez-Menéndez, L., Rubio OrDóÑEz, A., Cuesta, A. \& Gerdes, A. 2014. Origin and provenance of igneous clasts from late $\mathrm{Pa}$ laeozoic conglomerate formations (Del Ratón and El Planchón) in the Andean Precordillera of San Juan, Argentina. Journal of Iberian Geology 40(2), 261-82, doi:10.5209/rev_JIGE.2014.v40.n2.45298.

García de Madinabeitia, S., SÁnchez Lorda, M. E. \& GIL IBARGUCHI, J. I. 2008. Simultaneous determination of major to ultratrace elements in geological samples by fusion-dissolution and inductively coupled plasma mass spectrometry techniques. Analytica Chimica Acta 625(2), 117-30, doi.org/10.1016/j.aca.2008.07.024.

Gigliuto, L. G., Ouazani-Touhami, A., Puglisi, D., Puglisi, G. \& NaJiB Zaghloul, M. 2004. Petrography and geochemistry of granitoid pebbles from the Oligocene-Miocene deposits of the internal Rifian Chain (Morocco): a possible new hypothesis of provenance and paleogeographical implications. Geologica Carpathica 55(3), 261-72.

HARKer, A. 1909. The Natural History of Igneous Rocks. New York: Macmillan.

Ietto, A., Pescatore, T. \& Cocco, E. 1965. Il flysch mesozoico del Cilento occidentale. Bollettino della Società dei Naturalisti in Napoli 74, 396-402.

Ioppolo, S. \& Puglisi, G. 1980. I graniti peraluminosi di Delianuova (Aspromonte, Calabria Meridionale). Bollettino della Società Geologica Italiana 99, 269-80.

Ippolito, F., D’Argenio, B., Pescatore, T. \& Scandone, P. 1973. Unità stratigrafico-strutturali e schema tettonico dell'Appennino Meridionale. Istituto di Geologia e
Geofisica Università di Napoli, Pubblicazione n. 15, p. 38.

IRvine, T. N. \& Baragar, W. R. 1971. A guide to the chemical classification of the common igneous rocks. Canadian Journal of Earth Sciences 8, 523-48.

JACOBACCI, A. \& MARTELLI, G. 1967. Note illustrative alla carta geologica d'Italia scala 1:100.000. Foglio $174-$ Ariano Irpino. Servizio Geologico d'Italia, Roma, 65 pp.

JANOUŠEK, V., FARrow, C. M. \& ERban, V. 2006. Interpretation of whole-rock geochemical data in igneous geochemistry: introducing Geochemical Data Toolkit (GCDkit). Journal of Petrology 47(6),1255-9, doi:10.1093/petrology/eg1013.

Kastens, K. \& Mascle, J. (eds) 1990. The geological evolution of the Tyrrhenian Sea: An introduction to the scientific results of ODP Leg 107. In Proceedings of the ODP, Scientific Results, pp. 3-26. Texas A \& M University, Ocean Drilling Program, College Station, TX, USA, 3-26.

KnotT, S. D. 1987. The Liguride Complex of Southern Italy - a Cretaceous to Paleogene accretionary wedge. Tectonophysics 142, 217-26, doi:10.1016/00401951(87)90124-7.

Lammerer, B., Gebrande, H., Lüschen, E. \& Veselá, P. 2008. A crustal-scale cross-section through the Tauern Window (eastern Alps) from geophysical and geological data. In Tectonic Aspects of the Alpine-DinarideCarpathian System (eds S. Siegesmund, B. Fügenschuh \& N. Froitzheim), pp. 219-29. Geological Society of London, Special Publication no. 298.

Lardeaux, J. M., Schwartz, S., Tricart, P., Paul, A., Guillot, S., Béthoux, N. \& Masson, F. 2006. A crustal-scale cross-section of the south-western Alps combining geophysical and geological imagery. Terra Nova 18, 412-422, doi:10.1111/j.13653121.2006.00706.x.

LAUBSCHER, H. \& BeRNOULli, D. 1977. Mediterranean and Tethys. In: The Ocean Basins and Margins (eds A. E. M. Nairn, W. H. Kanes, F. G. Stehli), pp. 1-28. New York: Springer.

Le Bas, M. J., Le Maitre, R. W., Streckeisen, A. \& ZANETTIN, B. 1986. A chemical classification of volcanic rocks based on the Total Alkali Silica Diagram. Journal of Petrology 27(3), 745-50, doi:10.1093/petrology/27.3.745.

Lombardi, G., Cozzupoli, D. \& Nicoletti, M. 1974. Notizie geopetrografiche e dati sulla cronologia K-Ar del vulcanesimo tardo-paleozoico sardo. Periodico di Mineralogia 43, 221-312.

Lowe, D. R. 1982. Sediment gravity flows: II. Depositional models with special reference to the deposits of high-density turbidity currents. Journal of Sedimentary Petrology 52, 279-97, doi: 10.1306/212F7F31-2B2411D7-8648000102C1865D.

Maggi, M., Cianfarra, P. \& Salvini, F. 2016. Erosion by tectonic carving in the Concordia Subglacial Fault Zone, East Antarctica. Earth and Planetary Science Letters 433, 99-108, doi:10.1016/j.epsl.2015.10.045.

Maggi, M., Rossetti, F., Corfu, F., Theye, T., Andersen, T. B. \& FACCENNA, C. 2012. Clinopyroxene-rutile phyllonites from the East Tenda Shear Zone (Alpine Corsica, France): pressure-temperature-time constraints to the Alpine reworking of Variscan Corsica. Journal of the Geological Society, London 169, 723-32, doi:10.1144/jgs2011-120.

Maggi, M., Rossetti, F., Ranalli, G. \& Theye, T. 2014. Feedback between fluid infiltration and rheology along 
a regional ductile-to-brittle shear zone: The East Tenda Shear Zone (Alpine Corsica). Tectonics 33, 253-80, doi: 10.1002/2013TC003370.

Martelli, L. \& NARDI, G. 2005. Note illustrative della carta geologica d'Italia scala 1:50.000, foglio 503 - Vallo della Lucania. Firenze: Società Elaborazioni Cartografiche $86 \mathrm{pp}$.

Martini, I., Arragoni, S., Aldinucci, M., Foresi, L. M., BAmbini, A. M. \& SANDrelli, F. 2013. Detection of detached forced-regressive nearshore wedeges: a case study from the central-southern Siena Basin (Northern Apennines, Italy). International Journal of Earth Science 102, 1467-89, doi:10.1007/s00531-013-0876-6.

Martini, I., Sagri, M. \& Colella, A. 2001. NeogeneQuaternary basins of the inner Apennines and Calabrian arc. In: Anatomy of an Orogen, the Apennines and Adjacent Mediterranean Basins (eds G. B. Vai, \& I. P. Martini), 375-400. Dordrecht: Kluwer Academic Publishers, doi: 10.1007/978-94-015-9829-3_22.

Mazzeo, F. C., De Vita, P., Aulinas, M., Arienzo, I., Cirillo, G., Iovine, R. S. \& Sparice, D. 2016. New constraints on the origin of the ophiolitic rocks within sin-orogenic turbiditic sequences at Cilento region (southern Italy). Geologica Acta 14(3), 299-314, doi:10.1344/GeologicaActa2016.14.3.6.

Messina, A., Russo, S. \& Lima, A. 1993. Detailed exploration geochemistry of specialized granites of the Sila Batholith (Calabria, Italy) by fluid inclusion constraints on fluid source. Bollettino della Società Geologica Italiana 112, 999-1020.

Messina, A., Russo, S., Perrone, V. \& Giacobbe, A. 1991. Calc-alkaline Late Variscan two mica-cordierite-Alsilicate-bearing intrusions of the Sila batholith (Northern Sector of the Calabrian-Peloritan Arc - Italy). Bollettino della Società Geologica Italiana 110, 365389.

Messina, A., Russo, S., Stagno, F. \& Calandra, M. 1988. Petrochemistry and mineralogy of Monte Cacciagrande granite Stilo Unit (Calabrian-Peloritan Arc, Southern Italy). Rendiconti Società Italiana di Mineralogia e Petrologia 43(2), 569-86.

Minzoni, N. 1995. The geodynamic evolution of the Sardinian-Corsican and Calabrian-Peloritan Hercynian massifs. In: Basement Tectonics 10 (eds R. W. Ojakangas, A. B. Dickas \& J. C. Green), pp. 333-43. Proceedings of the International Conferences on Basement Tectonics held in Duluth (Minnesota, USA), vol. 4.. Dordrecht: Springer, doi:10.1007/978-94-017-08319_31.

MiYASHIRO, A. 1974. Volcanic rock series in island arcs and active continental margins. American Journal of Science 274, 321-55.

Molli, G. 2008. Northern Apennine - Corsica orogenic system: an updated overview. In Tectonic Aspects of the Alpine-Dinaride-Carpathian System (eds S. Siegesmund, B. Fügenschuh \& N. Froitzheim), pp. 413-42. Geological Society of London, Special Publication no. 298, doi:10.1144/SP298.19.

Monaco, C. \& Tortorici, L. 1995. Tectonic role of ophiolite-bearing terranes in the development of the Southern Apennines orogenic belt. Terra Nova 7(2), 153-60, doi:10.1111/j.1365-3121.1195.tb00684.x.

Mostardini, F. \& Merlini, S. 1986. Appennino CentroMeridionale. Sezioni geologiche e proposta di modello strutturale. Memorie Della Società Geologica Italiana 35, 177-202.

Nemec, W. \& Steel, R. J. 1984. Alluvial and coastal conglomerates: their significant features and some com- ments on gravelly mass-flow deposits. In: Sedimentology of Gravels and Conglomerates (eds E. H. Koster \& R. J. Steel), pp. 1-30. Canadian Society of Petroleum Geologists, Memoir no. 10.

Nemec, W. \& Steel, R. J. 1988. Fan Deltas - Sedimentology and Tectonic Settings. London: Blackie.

Nemec, W., Steel, R. J., Porebski, S. J. \& Spinnangr, A. 1984. Domba Conglomerate, Devonian, Norway: process and lateral variability in a mass-flow dominated, lacustrine fan-delta. In: Sedimentology of Gravels and Conglomerates (eds E. H. Koster \& R. J. Steel), pp. 295-320. Canadian Society of Petroleum Geologists, Memoir no. 10.

Oggiano, G. \& Di PisA, A. 2001. Introduction to the Sardinia geologic evolution. Rendiconti Seminario $\mathrm{Fa}$ coltà Scienze Università Cagliari 71(2), 5-11.

Oggiano, G., Funedda, A., Carmignani, L. \& Pasci, S. 2011. Reply to: Discussion on "The Sardinia-Corsica microplate and its role in the Northern Apennine Geodynamics: new insights from the Tertiary intraplate strike-slip tectonics of Sardinia" by I. Dieni \& F. Massari. Italian Journal of Geosciences 130(1), 139-44, doi:10.3301/IJG.2010.26.

OGniben, L. 1969. Schema introduttivo alla geologia del confine calabro-lucano. Memorie della Società Geologica Italiana 8, 453-763.

OGniben, L. 1972. Gli elementi strutturali della regione appenninica. Memorie Istituto Geologia Mineraria, Padova 29, 1-29.

OrszaG-Sperber, F. 2006. Changing perspectives in the concept of "Lago-Mare" in Mediterranean Late Miocene evolution. Sedimentary Geology 188-9, 259-77, doi: 10.1016/j.sedgeo.2006.03.008.

Pantosti, D. \& Velonì, M. 1986. Tettonica recente nell'Isola di Zannone e nella piattaforma circostante (Arcipelago Pontino, Italia Centrale). Memorie della Società Geologica Italiana 35, 623-29.

Parotto, M. \& Praturlon, A. 1975. Geological Summary of the Central Apennines. Quaderni de "La Ricerca Scientifica" 90. Roma: Consiglio Nazionale delle Ricerche, 257-311.

Passeri, L., Ciarapica, G., Reggiani, L. \& Rutledge, D. C. 2014. The significance of Longobucco Unit (Calabria-Peloritani Arc) in the evolution of the Ionian and Alpine Oceans. Bollettino della Società Geologica Italiana 133(2), 249-70, doi:10.3301/IJG.2014.07.

Patacca, E., Sartori, R. \& Scandone, P. 1990. Tyrrhenian basin and Apenninic arcs: kinematics relations since Late Tortonian times. Memorie della Società Geologica Italiana 45, 425-51.

Patacca, E. \& Scandone, P. 2001. Late thrust propagation and sedimentary response in the thrust-belt - foredeep system of the Southern Apennines (Pliocene - Pleistocene). In: Anatomy of an Orogen, the Apennines and Adjacent Mediterranean Basins (eds G. B. Vai \& I. P. Martini), pp. 401-40. Dordrecht: Kluwer Academic Publishers.

Patacca, E. \& Scandone, P. 2007. Geology of the Southern Apennines. In: Results of the CROP Project, Sub-project CROP-04 Southern Apennines (Italy) (eds A. Mazzotti, E. Patacca \& P. Scandone). Bollettino della Società Geologica Italiana 7, 75119.

Peccerillo, A. \& Donati, C. 2003. The Tuscan Magmatic Province. Periodico di Mineralogia 72, 27-39.

Pescatore, T. \& Ortolani, F. 1973. Schema tettonico dell'Appennino campano-lucano. Bollettino della Società Geologica Italiana 92, 453-472. 
Poli, G., Ghezzo, C. \& Conticelli, S. 1989. Geochemistry of granitic rocks from the Hercynian Sardinia-Corsica batholith: Implication for magma genesis. Lithos 23, 247-66, doi:10.1016/0024-4937(89)90038-8.

Reading, H. G. 1996. Sedimentary Environments: Processes, Facies and Stratigraphy, 3rd edition. Oxford, UK: Wiley-Blackwell, 704 pp.

Recio, C., Fallick, A. E., Ugidos, J. M. \& Stephens, W. E. 1997. Characterization of multiple fluid-granite interaction processes in the episyenites of Avila-Bejar, Central Iberian Massif, Spain. Chemical Geology 143, 127-44, doi:10.1016/S0009-2541(97)00106-X.

Rossetti, F., Glodny, J., Theye, T. \& Maggi, M. 2015. Pressure-temperature-deformation-time of the ductile Alpine shearing in Corsica: from orogenic construction to collapse. Lithos 218-9, 99-116, doi:10.1016/j.lithos.2015.01.011.

Rubio Ordóñez, A., Barba, P., Cuesta, A., Gallastegui, G., Suárez, O., Ugidos, J. M., \& Valladares, M. I. 2004. Los cantos volcánicos del conglomerado basal de la Fm. Herrería: Evidencias de un Volcanismo Neoproterozoico en la base del Cámbrico. Geogaceta 36, $11-14$.

SAcco, F. 1910. L'Appennino Meridionale (studio geologico sintetico). Bollettino della Società Geologica Italiana 29, 287-368.

SAlvini, F. \& VitTori, E. 1982. Analisi strutturale della linea Olevano-Antrodoco-Posta (Ancona-Anzio Auct.): metodologia di studio delle deformazioni fragili e presentazione del tratto meridionale. Memorie della Società Geologica Italiana 24, 337-55.

Santantonio, M. \& Teale, C. T. 1987. An example of the use of detrital episodes in elucidating complex basin histories: the Caloveto and Longobucco Groups of NE Calabria, S Italy. In: Marine Clastic Sedimentology (eds J. K. Legget \& G. G. Zuffa), pp. 62-74. Dordrecht: Graham \& Trotman, doi:10.1007/978-94-009-3241-8_3.

Sartori, R., Mascle, G. \& Amaudric du Chaffaut, S. 1987. A review of circum-tyrrhenian regional geology. In: Proceedings of the Ocean Drilling Program 107, Initial Report (eds K. A. Kastens, J. Mascle, C. Auroux et al.), pp. 37-63. Texas A \& M University, Ocean Drilling Program, College Station, Texas, USA.

Sartori, R., Torelli, L., Zitellini, N., Carrara, G., Magaldi, M. \& Mussoni, P. 2004. Crustal features along a W-E Tyrrhenian transect from Sardinia to Campania margins (Central Mediterranean). Tectonophysics 383, 171-92, doi:10.1016/j.tecto.2004.02.008.

SCANDONE, P. 1967. Studi di geologia lucana: la serie calcareo-silico-marnosa. Bollettino della Società dei Naturalisti Napoli 74, 311-6.

SCANDONE, P. 1975. Triassic seaways and the Jurassic Tethys Ocean in the central Mediterranean area. Nature 256/5513, 117-9.

Schmid, S. M., Pfiffner, O. A., Froitzheim, N., Schönborn, G. \& Kissling, E. 1996. Geophysicalgeological transect and tectonic evolution of the Swiss-Italian Alps. Tectonics 15(5), 1036-64, doi:10.1029/96TC00433.

SelLI, R. 1957. Sulla trasgressione del Miocene nell'Italia meridionale. Giornale di Geologia 26, 1-54.

SELLI, R. 1962. Il Paleogene nel quadro dell'Italia meridionale. Memorie della Società Geologica Italiana 3, 737-90.

SGN 1939. Carta Geologica d'Italia alla scala 1:100.000, Foglio 151 - Alatri. Roma: Istituto Poligrafico e Zecca dello Stato.
SGN 1963. Carta Geologica d'Italia alla scala 1:100.000, Foglio 174 - Ariano Irpino. Roma: Istituto Poligrafico e Zecca dello Stato.

SGN 1967. Carta Geologica d'Italia alla scala 1:100.000, Foglio 159 - Frosinone. Roma: Istituto Poligrafico e Zecca dello Stato.

SGN 1969. Carta Geologica d'Italia alla scala 1:100.000, Foglio 209 - Vallo della Lucania. Roma: Istituto Poligrafico e Zecca dello Stato.

SGN 1970. Carta Geologica d'Italia alla scala 1:100.000, Foglio 147 - Lanciano. Roma: Istituto Poligrafico e Zecca dello Stato.

SGN 1975. Carta Geologica d'Italia alla scala 1:50.000, Foglio 389 - Anagni. Roma: Istituto Poligrafico e Zecca dello Stato.

SGN 2007. Carta Geologica d'Italia alla scala 1:50.000, Foglio 433 - Ariano Irpino. Roma: Consiglio Nazionale delle Ricerche.

Sgrosso, I. 1986. Criteri ed elementi per una ricostruzione paleogeografica delle zone esterne dell'Appennino Centro-Meridionale. Memorie della Società Geologica Italiana 35, 203-19.

Speranza, F., Villa, I. M., Sagnotti, L., Florindo, F., Cosentino, D., Cipollari, P. \& Mattei, M. 2002. Age of the Corsica-Sardinia rotation and Liguro-Provençal Basin spreading: new paleomagnetic and $\mathrm{Ar} / \mathrm{Ar}$ evidence. Tectonophysics 347, 231-51, doi:10.1016/S00401951(02)00031-8.

Streckeisen, A. 1974. Classification and nomenclature of plutonic rocks. Recommendations of the IUGS Subcommission on the systematics of igneous rocks. Geologische Rundschau 63(2), 773-86, doi:10.1007/BF01820841.

TAYlOR, H. P. \& TuRI, B. 1976. High- ${ }^{18} \mathrm{O}$ igneous rocks from the Tuscan Magmatic Province, Italy. Contributions to Mineralogy and Petrology 55, 33-54.

Torre, M., Di Nocera, S. \& Matano, F. 2007. Note illustrative della carta geologica d'Italia alla scala 1:50.000. Foglio 433 - Ariano Irpino. Roma: ISPRA, $140 \mathrm{pp}$.

Torrente, M. M., Civile, D., Martino, C. \& Milia, A. 2000. Assetto strutturale ed evoluzione tettonica dell'area di Monte Vesole - Monte Chianello (Cilento, Appennino meridionale). Bollettino della Società Geologica Italiana 119, 733-47.

Tortorici, L., Catalano, S. \& Monaco, C. 2009. Ophiolite-bearing mélanges in southern Italy. Geological Journal 44, 153-166.

Traversa, G. \& VACCARO, C. 1992. REE distribution in the Late Hercynian dykes from Sardinia. In: Contributions to the Geology of Italy with Special Regard to the Paleozoic Basements. A Volume Dedicated to Tommaso Cocozza (eds L. Carmignani \& F. P. Sassi), pp. 215-26. IGCP no 276, Newsletter vol. 5, Siena 1992, 215-26.

TRÜMPY, R. 1973. The timing of orogenic events in the Central Alps. In: Gravity and Tectonics (eds K. A. De Jong \& R. Scholten), pp. 229-51. New York: Wiley.

VAI, G. B. 2001. Basement and early (pre-Alpine) history. In: Anatomy of an Orogen, the Apennines and Adjacent Mediterranean Basins (eds G. B. Vai \& I. P. Martini), pp. 121-50. Dordrecht: Kluwer Academic Publishers.

Vai, G. B. \& Martini, I. P. 2001. Anatomy of an Orogen, the Apennines and Adjacent Mediterranean Basins. Dordrecht: Kluwer Academic Publishers, $632 \mathrm{pp}$. 
VALENTE, A. 1991. Caratteri sedimentologici di una successione torbiditica nel Cilento Orientale (Appennino Meridionale). Memorie della Società Geologica Italiana 47, 191-96.

Vezzani, L., Festa, A. \& Ghisetti, F. C. 2010. Geology and Tectonic Evolution of the Central-Southern Apennines, Italy. Boulder, Colorado: Geological Society of America, Special Paper no. 469, $58 \mathrm{pp}$.

Viola, C. 1898. Osservazioni geologiche fatte sui Monti Sublacensi nel 1897. Bollettino della Società Geologica Italiana 17, 272-84.
Whitney, D. L. \& Evans, B. W. 2010. Abbreviations for names of rock-forming minerals. American Mineralogist 95, 185-87, doi:10.2138/am.2010.3371.

Winchester, J. H. \& Floyd, P. A. 1977. Geochemical discrimination of different magma series and their differentiation products using immobile elements. Chemical Geology 20, 325-43, doi:10.1016/00092541(77)90057-2.

ZufFA, G. C. 1980. Hybrid arenites: Their composition and classification. Journal of Sedimentary Petrology 50, 21-30, doi:10.1306/212F7950-2B24-11D7$8648000102 \mathrm{C} 1865 \mathrm{D}$. 